\title{
Establishment of a Cell Suspension Culture of Eysenhardtia platycarpa: Phytochemical Screening of Extracts and Evaluation of Antifungal Activity
}

\author{
Antonio Bernabé-Antonio ${ }^{1, *(1)}$, Alejandro Sánchez-Sánchez ${ }^{1}$, Antonio Romero-Estrada ${ }^{1}$, \\ Juan Carlos Meza-Contreras ${ }^{1}$, José Antonio Silva-Guzmán ${ }^{1}$, Francisco Javier Fuentes-Talavera ${ }^{1}$,

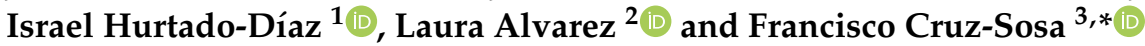

1 Departamento de Madera, Celulosa y Papel, Centro Universitario de Ciencias Exactas e Ingenierías, Universidad de Guadalajara, Km 15.5 Carretera Guadalajara-Nogales, Col. Las Agujas, Zapopan 45100, Jalisco, Mexico; alexcucba.bio@gmail.com (A.S.-S.); are@uaem.mx (A.R.-E.); jcmezac@academicos.udg.mx (J.C.M.-C.); jasilva@dmcyp.cucei.udg.mx (J.A.S.-G.); francisco.fuentes@academicos.udg.mx (F.J.F.-T.); ihurtado@uaem.mx (I.H.-D.)

2 Centro de Investigaciones Químicas-IICBA, Universidad Autónoma del Estado de Morelos, Av. Universidad No. 1001, Col. Chamilpa, Cuernavaca 62209, Morelos, Mexico; lalvarez@uaem.mx

3 Departamento de Biotecnología, División de Ciencias Biológicas y de la Salud, Universidad Autónoma Metropolitana-Unidad Iztapalapa, San Rafael Atlixco 186, Col. Vicentina, Ciudad de México 09340, Mexico

check for updates

Citation: Bernabé-Antonio, A.

Sánchez-Sánchez, A.;

Romero-Estrada, A.; Meza-Contreras, J.C.; Silva-Guzmán, J.A.;

Fuentes-Talavera, F.J.; Hurtado-Díaz,

I.; Alvarez, L.; Cruz-Sosa, F.

Establishment of a Cell Suspension

Culture of Eysenhardtia platycarpa:

Phytochemical Screening of Extracts and Evaluation of Antifungal Activity. Plants 2021, 10, 414. https://doi.org/ $10.3390 /$ plants10020414

Academic Editors: Sandra Gonçalves, Anabela Romano and Sofia Caretto

Received: 8 December 2020

Accepted: 19 February 2021

Published: 23 February 2021

Publisher's Note: MDPI stays neutral with regard to jurisdictional claims in published maps and institutional affiliations.

Copyright: (c) 2021 by the authors. Licensee MDPI, Basel, Switzerland. This article is an open access article distributed under the terms and conditions of the Creative Commons Attribution (CC BY) license (https:/ / creativecommons.org/licenses/by/ $4.0 /)$.
* Correspondence: bernabe_aa@hotmail.com (A.B.-A.); cuhp@xanum.uam.mx (F.C.-S.)

\begin{abstract}
Eysenhardtia platycarpa (Fabaceae) is a medicinal plant used in Mexico. Biotechnological studies of its use are lacking. The objective of this work was to establish a cell suspension culture (CSC) of E. platycarpa, determine the phytochemical constituents by spectrophotometric and gas chromatography-mass spectrometry (GC-MS) methods, evaluate its antifungal activity, and compare them with the intact plant. Friable callus and CSC were established with $2 \mathrm{mg} / \mathrm{L}$ 1-naphthaleneacetic acid plus $0.1 \mathrm{mg} / \mathrm{L}$ kinetin. The highest total phenolics of CSC was $15.6 \mathrm{mg}$ gallic acid equivalents (GAE)/g dry weight and the total flavonoids content ranged from 56.2 to $104.1 \mu \mathrm{g}$ quercetin equivalents (QE)/g dry weight. The GC-MS analysis showed that the dichloromethane extracts of CSC, sapwood, and heartwood have a high amount of hexadecanoic acid (22.3-35.3\%) and steroids (13.5-14.7\%). Heartwood and sapwood defatted hexane extracts have the highest amount of stigmasterol $(\sim 23.4 \%)$ and $\beta$-sitosterol ( 43\%), and leaf extracts presented $\beta$-amyrin (16.3\%). Methanolic leaf extracts showed mostly sugars and some polyols, mainly D-pinitol (74.3\%). Compared with the intact plant, dichloromethane and fatty hexane extracts of CSC exhibited percentages of inhibition higher for Sclerotium cepivorum: $71.5 \%$ and $62.0 \%$, respectively. The maximum inhibition for Rhizoctonia solani was with fatty hexane extracts of the sapwood (51.4\%). Our study suggests that CSC extracts could be used as a possible complementary alternative to synthetic fungicides.
\end{abstract}

Keywords: plant in vitro culture; plant extracts; gas chromatography; hexadecanoic acid; antifungal activity

\section{Introduction}

For centuries, plants have been an important source of natural products for humans; they have been used as flavorings and condiments and for treating health disorders and preventing diseases, including epidemics [1]. Plant products have formed the basis for many useful pharmaceuticals and agrochemicals, and through their rational use, plants can be a potential alternative for obtaining extracts or bioactive compounds to control several diseases in both humans and crops [2,3]. However, many of these extracts or compounds are isolated from wild plants, whose collection generally has a negative impact on the environment [4]. Furthermore, agricultural soil is increasingly limited and, in the future, the production of plants in the field will not be feasible. Although plants can be cultivated 
in the field, many of them need several years to be harvested, and in many cases, the yields of bioactive compounds and biological activity of cultivated or wild plants are lower compared with cultured plant cells [5,6]. Plant cell culture is a biotechnological tool that has the potential to accelerate the production of natural products in a controlled environment; in addition, cell culture provides a renewable source of natural products, since plant cell culture can be produced and harvested at all times of the year [3,4].

In this regard, Eysenhardtia platycarpa (Fabaceae) is a wild plant extensively exploited as firewood, fodder, or to manufacture utensils and furniture such as "equipales" and fences; in addition, in traditional Mexican medicine, an infusion prepared from the wood is used against kidney and gallbladder diseases [7-9]. All these uses, as well as forest fires, are causing a decline in wild populations. Among the few existing investigations on E. platycarpa, all of them have used the wild plant as a source of pharmacological studies [10]. For instance, flavonoids with cytotoxic and antibacterial activity were isolated from methanolic extracts of branches and leaves of E. platycarpa [11]. The in vivo antiinflammatory activity of flavones isolated from the leaves has also been reported [12]. However, no effort has been made to carry out studies aimed at the sustainable use of this species. In a biotechnological study developed for Eysenhardtia polystachya (a closely related species), the antifungal activity of cell suspension culture extracts was reported against R. solani and S. cepivorum [13]. To our knowledge, however, there are no reports involving biotechnological studies of E. platycarpa. Therefore, it is necessary to look for biotechnological techniques that allow us to obtain bioactive extracts while preserving natural diversity and the environment.

The main aim of this study was to establish a cell suspension culture from E. platycarpa internodal segments. We also performed the phytochemical characterization of the CSC and intact plant extracts through the spectrophotometric quantification of total phenolics and flavonoids content and, by GC-MS analysis, the volatile natural compounds or silylated derivatives were determined. Furthermore, the biological activity of the extracts against the phytopathogenic fungi Rhizoctonia solani and Sclerotium cepivorum was explored. As a hypothesis, we expected that the E. platycarpa extracts produced by cell suspension cultures would present comparable antifungal activity with respect to extracts from the wild plant, as well as the compounds identified by GC-MS.

\section{Results and Discussion}

\subsection{Obtaining Plantlets and Callus Induction}

In vitro cultures of Eysenhardtia platycarpa are shown in Figure 1. The seed germination rate was $98 \%$ at 10 days of culture and no microbial contamination was observed. The in vitro plants grew easily without plant growth regulators after being transferred to 1-L jars (Figure 1A).

In other Fabaceae species such as Prosopis laevigata, the in vitro germination can occur after 3-7 days in mechanically scarified seeds [14,15]. Regarding callus induction, a suitable response of callus formation was exhibited on internodal segments after 15 days of culture (Figure 1B). All treatments had percentages of callus induction greater than $50.0 \%$, regardless of the type of auxins (NAA: naphthaleneacetic acid; 2,4-D: 2,4-dichlorophenoxyacetic acid) or kinetin (KIN) as a cytokinin (Table 1).

The control (PGRs-free) showed callus formation in 50\% of segments, but exhibited scarce growth, showed a brown color, and later died. It is possible that E. platycarpa contains auxin in leaves and stems, which may explain why, even in the control treatment, the segments formed a callus. In fact, it has been reported that indoleacetic acid (IAA) occurs naturally in plants, mainly in young leaves or seeds [16,17]. Moreover, it is known that levels of naturally occurring auxin in explant tissues depend on the mother plant from which the explants were taken [18]. 


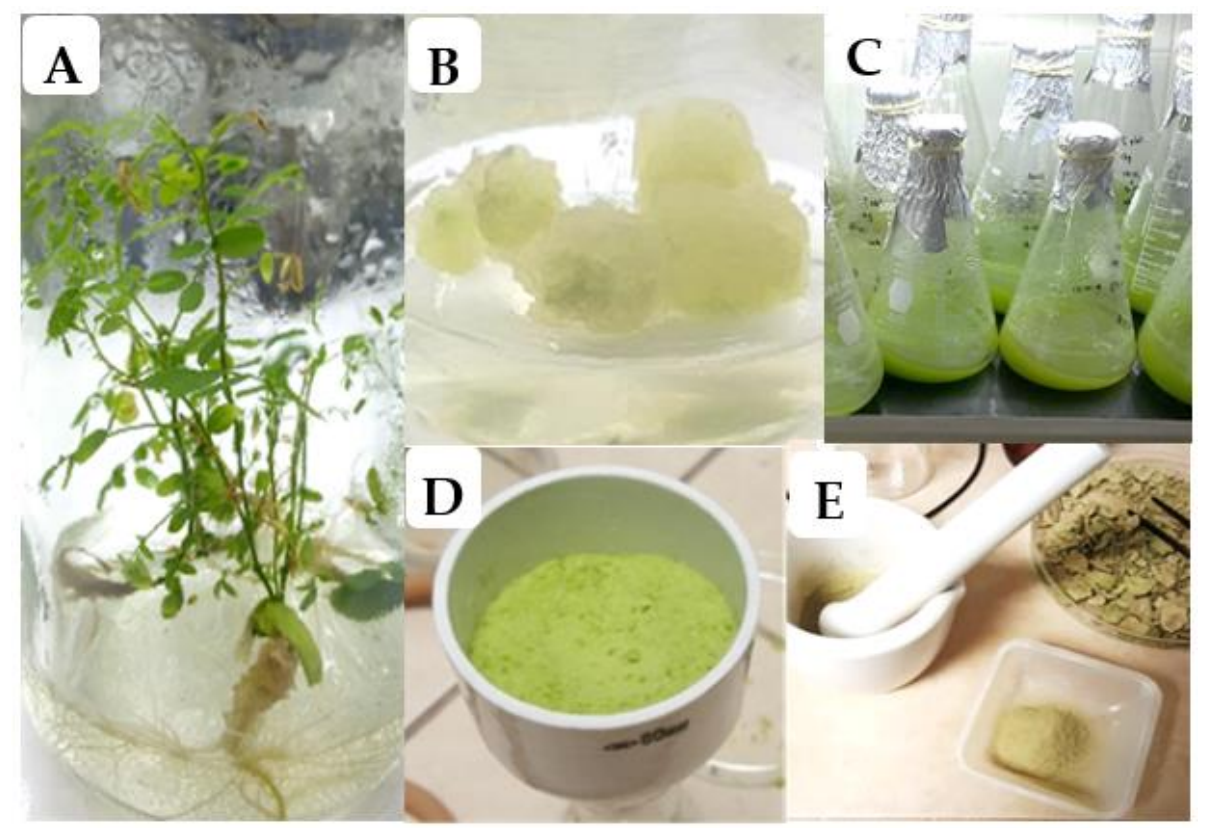

Figure 1. In vitro cultures of Eysenhardtia platycarpa. (A) Plantlets grown in MS culture medium without plant growth regulators; (B) callus production at 15 days of culture; (C) cell suspension cultures with $2 \mathrm{mg} / \mathrm{L}$ NAA and $0.5 \mathrm{mg} / \mathrm{L} \mathrm{KIN;} \mathrm{(D)} \mathrm{fresh} \mathrm{biomass} \mathrm{harvested} \mathrm{after} 12$ days of culture; (E) dried biomass used for obtaining extracts.

Table 1. Percentage of internodal explants of Eysenhardtia platycarpa inducing callus in Murashigue and Skoog (MS) culture medium after 30 days of culture.

\begin{tabular}{|c|c|c|c|c|c|}
\hline \multicolumn{2}{|c|}{ PGRs (mg/L) } & \multirow{2}{*}{ Callus Induction (\%) } & \multicolumn{2}{|c|}{ PGRs (mg/L) } & \multirow{2}{*}{ Callus Induction (\%) } \\
\hline $2,4-\mathrm{D}$ & KIN & & NAA & KIN & \\
\hline 0.0 & 0.0 & $50.0 \pm 0.0^{b}$ & 0.0 & 0.0 & $50 \pm 0.0^{c}$ \\
\hline 0.0 & 0.1 & $66.7 \pm 7.2^{\mathrm{ab}}$ & 0.0 & 0.1 & $58.3 \pm 19.1^{b c}$ \\
\hline 0.0 & 1.0 & $93.8 \pm 6.3^{\mathrm{a}}$ & 0.0 & 1.0 & $100.0 \pm 0.0^{\mathrm{a}}$ \\
\hline 0.0 & 2.0 & $75.0 \pm 10.2^{\mathrm{ab}}$ & 0.0 & 2.0 & $100.0 \pm 0.0^{\mathrm{a}}$ \\
\hline 0.1 & 0.0 & $81.3 \pm 12.0^{\mathrm{ab}}$ & 0.1 & 0.0 & $75.0 \pm 10.2^{\mathrm{ab}}$ \\
\hline 0.1 & 0.1 & $81.3 \pm 12.0^{\mathrm{ab}}$ & 0.1 & 0.1 & $93.8 \pm 6.3^{\mathrm{a}}$ \\
\hline 0.1 & 1.0 & $93.8 \pm 6.3^{a}$ & 0.1 & 1.0 & $93.8 \pm 6.3^{\mathrm{a}}$ \\
\hline 0.1 & 2.0 & $87.5 \pm 12.5^{\mathrm{ab}}$ & 0.1 & 2.0 & $100.0 \pm 0.0^{\mathrm{a}}$ \\
\hline 1.0 & 0.0 & $75.0 \pm 10.2^{a b}$ & 1.0 & 0.0 & $93.8 \pm 6.3^{\mathrm{a}}$ \\
\hline 1.0 & 0.1 & $100.0 \pm 0.0^{\mathrm{a}}$ & 1.0 & 0.1 & $100.0 \pm 0.0^{\mathrm{a}}$ \\
\hline 1.0 & 1.0 & $83.3 \pm 14.4^{\mathrm{ab}}$ & 1.0 & 1.0 & $100.0 \pm 0.0^{\mathrm{a}}$ \\
\hline 1.0 & 2.0 & $100.0 \pm 0.0^{\mathrm{a}}$ & 1.0 & 2.0 & $100.0 \pm 0.0^{\mathrm{a}}$ \\
\hline 2.0 & 0.0 & $83.3 \pm 14.4^{\mathrm{ab}}$ & 2.0 & 0.0 & $100.0 \pm 0.0^{\mathrm{a}}$ \\
\hline 2.0 & 0.1 & $100.0 \pm 0.0^{\mathrm{a}}$ & 2.0 & 0.1 & $100.0 \pm 0.0^{\mathrm{a}}$ \\
\hline 2.0 & 1.0 & $91.7 \pm 7.2^{\mathrm{a}}$ & 2.0 & 1.0 & $100.0 \pm 0.0^{\mathrm{a}}$ \\
\hline 2.0 & 2.0 & $100.0 \pm 0.0^{\mathrm{a}}$ & 2.0 & 2.0 & $100.0 \pm 0.0^{\mathrm{a}}$ \\
\hline
\end{tabular}

PGRs: Plant growth regulators; 2,4-D: 2,4-dichlorophenoxyacetic acid; NAA: naphthaleneacetic acid; KIN: kinetin Values represent mean \pm standard deviation of four replicates per treatment in two repeated experiments. Means followed by the same letter in superscript in the same column are not significantly different $(p=0.05)$ according to Tukey's multiple range test.

In internodal segments of E. platycarpa, all evaluated treatments showed a positive effect on callus formation; $62.0 \%$ of the treatments with NAA and KIN formed a callus on $100 \%$ of the segments, mainly with NAA $(1.0$ or $2.0 \mathrm{mg} / \mathrm{L})$, regardless of KIN concentration. Calluses of this treatment were clear greenish and more friable in appearance than the other treatments; moreover, the calluses had homogeneous growth during all subcultures. The other treatments exhibited a smaller and semicompact callus, and most of them did 
not show growth. In the case of treatments with 2,4-D plus KIN, it was observed that, as the concentration of 2,4-D increased, there was a trend of an increased percentage of callus induction, and this generally occurred in the presence of KIN (Table 1). Similarly, treatments with 2,4-D (1.0 or $2.0 \mathrm{mg} / \mathrm{L})$ combined with KIN $(0.1$ or $2.0 \mathrm{mg} / \mathrm{L})$ had $100 \%$ of explants with calluses. The remaining treatments, including the control, showed calluses with percentages between $50.0 \%$ and $94.0 \%$. Calluses of the best treatments with both auxins, i.e., NAA (2.0 mg/L) with KIN (0.1 mg/L) or 2,4-D (2.0 mg/L) with KIN (0.1 mg/L), were subcultured periodically for six months to increase callus production.

In a study reported for E. polystachya, it was found that callus induction was variable, according to the PGRs, i.e., percentages of calluses between $65.6 \%$ and $98.4 \%$ in leaf explants were obtained with picloram (PIC) plus KIN, and from $64.1 \%$ to $100 \%$ with NAA plus KIN [13]. In studies carried out on P. laevigata, calluses from cotyledons, hypocotyls, and root explants were obtained with 2,4-D plus 6-benzylaminopurine (BAP) or KIN, in percentages of $28.0 \%$ to $100 \%$ [15]. This indicates that combining auxins and cytokinins plays an indispensable role in inducing and increasing the percentage of calluses [19].

\subsection{Cell Suspension Cultures}

\subsubsection{Growth Kinetics and Sucrose Consumption}

Calluses induced with NAA $(2.0 \mathrm{mg} / \mathrm{L})$ and KIN $(0.1 \mathrm{mg} / \mathrm{L})$ or $2,4-\mathrm{D}(2.0 \mathrm{mg} / \mathrm{L})$ and $\mathrm{KIN}(0.1 \mathrm{mg} / \mathrm{L})$ were the best treatments, showing friable characteristics, and were used to initiate the establishment of the cell suspension cultures (CSC) of E. platycarpa with the same plant growth regulators; however, during one month of culture, cells cultured in MS liquid medium with 2,4-D (2.0 mg/L) and KIN $(0.1 \mathrm{mg} / \mathrm{L})$ showed poor growth. Therefore, this treatment was discarded from the experiment. In contrast, cells cultured with NAA $(2.0 \mathrm{mg} / \mathrm{L})$ and KIN $(0.1 \mathrm{mg} / \mathrm{L})$ exhibited growth and an abundant accumulation of biomass (Figure $1 \mathrm{C}-\mathrm{E}$ ).

The growth kinetics of E. platycarpa was maintained for 18 days, during which it exhibited typical growth (Figure 2). According to the modified Gompertz model, the lag phase $[\lambda$ (days)] lasted 1.84 days. The exponential phase was six days (from day 2 to day 8 ). The stationary phase was observed between days 10 and 12; then, the senescence phase was gradually observed between days 14 and 18. In addition, the maximum accumulation of biomass dry weight (18.62 g/L DW) occurred after 10 days and the growth index was 5.35, obtaining a yield of $0.621 \mathrm{~g}$ dry biomass / $\mathrm{g}$ sucrose. By the natural logarithm of biomass, the specific growth rate $(\mu)$ was 0.24 days $^{-1}$ and the doubling time (td) was 2.92 days, while, using the modified Gompertz model, $\mu_{\max }$ was 0.25 days $^{-1}$ and $\mathrm{X}_{\max }$ was $19.6(\mathrm{~g} / \mathrm{L})$ with a correlation coefficient $(\mathrm{R})$ of 0.982 . This model has been reported for callus and cell suspension cultures of Jatropha curcas [20,21] and plant growth [22].

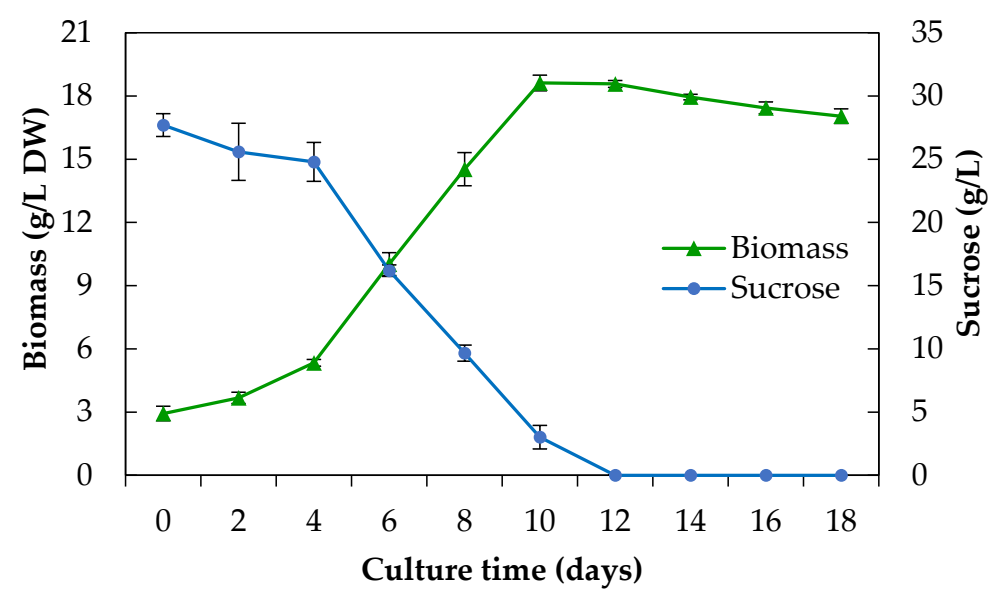

Figure 2. Growth kinetics and consumption of sucrose of a cell suspension culture of Eysenhardtia platycarpa for 18 days of culture. Values represent the mean \pm standard deviation of three replicates. 
During the adaptation phase of E. platycarpa, the amounts of sugars remained unchanged; however, in the exponential growth phase, there was a greater demand for sugars and, consequently, a higher production of biomass. From day 12, the sugars in the culture medium were almost completely consumed; this coincided with the stationary phase and the senescence phase. Sugars were not detected in the culture medium from day 12 ; however, it is likely that they are present in undetectable traces since the culture was not significantly affected in the senescence phase. This behavior has also been reported in other species such as $C$. brasiliense cell suspension cultures [23]. This may be due to the cells accumulating glucose and fructose and using them for metabolism [24]. In fact, GC-MS showed that the methanolic extract of cell suspension cultures had large amounts of disaccharides (more than $40 \%$ ).

In other species such as P. laevigata, the treatment with 2,4-D $(1.5 \mathrm{mg} / \mathrm{L})$ and $\mathrm{KIN}$ $(1.0 \mathrm{mg} / \mathrm{L})$ was the most suitable for establishing the CSC [15]. It is known from callus induction treatments on semisolid culture medium that these do not always adapt best to a liquid culture medium. For instance, P. laevigata leaf explants presented $100 \%$ of callus with trichlorophenoxyacetic acid (2,4,5-T, $1.28 \mathrm{mg} / \mathrm{L})$ with BAP $(1.13 \mathrm{mg} / \mathrm{L})$ or $2,4,5-\mathrm{T}$ $(1.28 \mathrm{mg} / \mathrm{L})$ with KIN $(1.08 \mathrm{mg} / \mathrm{L})$; however, only calluses containing KIN were suitable for establishing the CSC [14]. This indicates that, even among closely related species, the genotype is an influential factor in the response of cell cultures; moreover, the PGRs activity varies depending on the presence of transporter or receptor biosynthesized proteins in the explants, affecting in vitro culture development [25]. In another study conducted in E. polystachya, the maximum accumulation of dry biomass was $14 \mathrm{~g} / \mathrm{L}$ after 10 days of culture [13]. Biomass yield was lower than that reported in the present study for E. platycarpa at the same time. Studies conducted by Maldonado-Magaña et al. [15] in P. laevigata also found a dry biomass yield of $15.6 \mathrm{~g} / \mathrm{L}$ after 21 days of culture.

\subsubsection{Total Phenolics and Flavonoids Content}

It has been reported that phenolics (TPH), flavonoids (TFL), and other compounds of plant extracts are effective against phytopathogenic fungi [26,27]; therefore, it is desirable to quantify these groups of compounds in the extracts. Because the cell cultures were obtained from leaf explants, in this part, we made a comparison of TPH and TFL in cell suspension culture with leaf extracts. During culture time from E. platycarpa CSC, the TPH and TFL content had low variation (Figure 3).

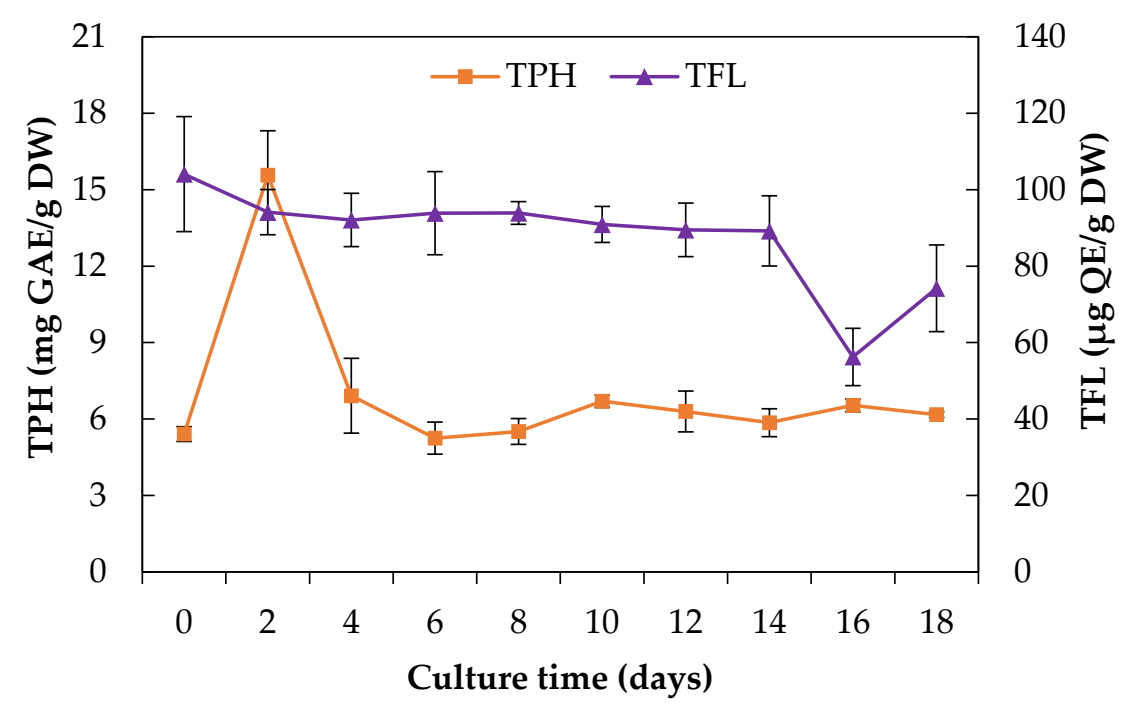

Figure 3. Production of total phenolics (TPH) and total flavonoids content (TFL) of a cell suspension culture of Eysenhardtia platycarpa over 18 days of culture. Values represent the mean \pm standard deviation of three replicates. 
During the adaptation phase of the culture, there was a notable increase in TPH (15.6 mg GAE/g DW) and then it remained constant, showing amounts between $5.2 \pm 0.63$ and $6.2 \mathrm{mg} \mathrm{GAE} / \mathrm{g}$ DW. Similar studies reported by Giri et al. [28] found a concentration of $10.17 \mathrm{mg} \mathrm{GAE} / \mathrm{g}$ DW after 28 days of culture of Habenaria edgeworthii. In this work, the TPH content of E. platycarpa leaves (11.77 mg GAE/g DW), was lower than cell suspension cultures on day 2; however, during this stage biomass production was low.

Regarding the TFL content, this also remained constant between days 2 and 14, except that day 0 had a value of $104.1 \mu \mathrm{g} \mathrm{QE} / \mathrm{g}$ DW. Near the end of the senescence phase (day 16), the TFL decreased to $56.2 \mu \mathrm{g} \mathrm{QE} / \mathrm{g}$ DW (Figure 3). On the other hand, low concentrations of TFL were also found in wild plant leaves $(88.2 \mu \mathrm{g} Q \mathrm{QE} / \mathrm{g} \mathrm{DW})$. In studies conducted in Saussurea medusa (Maxim) cell suspension cultures, the total flavonoid production was $607.8 \mathrm{mg} / \mathrm{L}$ after 15 days of culture [29]. In other species such as Clinacanthus nutans, large amounts of total polyphenols, phenolic acids, and flavonoids were found in in vitro plants compared with plants propagated by a stem cutting technique [6].

\subsection{Yield of Extracts of Cell Suspension Culture and Intact Plant}

In general, the methanolic and dichloromethane extracts exhibited the highest dry weight yields (Table 2). This coincides with what has been reported for related species such as E. polystachya, in which the highest yields were obtained with methanol and dichloromethane [30]. In Tectona grandis, yields of $2.9 \%, 2.3 \%$, and $3.6 \%$ were reported, through a sequential extraction with hexane, ethyl acetate, and methanol, respectively [31]. The methanolic extracts made of the heartwood of other Fabaceae (Caesalpinia platyloba and Lysiloma latisquum) also report the best yields of extracts using these solvents [32]. For E. platycarpa, the highest yield of the methanolic extract was $36.9 \%$ of the heartwood and $30.08 \%$ for the leaf (Table 2). Other studies carried out on branches of Severinia buxifolia also obtained a higher extract yield (33.2\%) using methanol [33]. In another study conducted on Caesalpinia sappan L., methanolic extracts of heartwood and leaf showed higher yields of $17.60 \%$ and $17.05 \%$, respectively [34]. This may be because plants contain large amounts of polar compounds such as proteins and carbohydrates [35]. High amounts of polyols may also have increased the yield of methanolic extracts [36].

Table 2. Yields of different solvent extraction of intact plant and cell suspension culture of Eysenhardtia platycarpa.

\begin{tabular}{ccccc}
\hline \multirow{2}{*}{ Extract } & \multicolumn{3}{c}{ Yield of Extract (\%) } \\
\cline { 2 - 5 } & Sapwood & Heartwood & Leaf & Cell Suspension Culture \\
\hline Fatty hexane & 4.0 & 6.7 & 8.0 & 0.1 \\
Defatted hexane & 5.0 & 8.6 & 10.1 & 0.5 \\
Dichloromethane & 15.0 & 22.8 & 20.0 & 2.4 \\
Methanolic & 21.1 & 36.9 & 30.8 & 23.8 \\
Total & 45.1 & 75.0 & 68.9 & 26.8 \\
\hline
\end{tabular}

\subsection{Compounds Identified by GC-MS}

The Fabaceae family produces a high diversity of bioactive compounds as defense against bacterial and fungi [37]. In the literature, few studies have reported on the determination of intact plant compounds of E. platycarpa; there are no studies on the phytochemical profile of its cell culture extracts.

By using gas chromatography-mass spectrometry (GC-MS) analysis, the phytochemical profile of the hexane, dichloromethane, and methanolic extracts of the cell suspension cultures, sapwood, heartwood, and leaves of the intact plant of E. platycarpa was determined (Tables 3 and 4). 
Table 3. Chemical constituents of the hexane extracts of cell suspension culture (CSC) and plant of Eysenhardtia platycarpa identified by GC-MS.

\begin{tabular}{|c|c|c|c|c|c|c|c|c|c|c|}
\hline \multirow{3}{*}{ Compound Name } & \multirow{3}{*}{ Chemical Formula } & \multirow{3}{*}{ RT (min) } & \multicolumn{8}{|c|}{ Abundance (\%) } \\
\hline & & & \multicolumn{4}{|c|}{ Fatty Hexane Extract } & \multicolumn{4}{|c|}{ Defatted Hexane Extract } \\
\hline & & & Sapwood & Heartwood & Leaf & CSC & Sapwood & Heartwood & Leaf & CSC \\
\hline \multicolumn{11}{|l|}{ Alkanes } \\
\hline Heptacosane & $\mathrm{C}_{27} \mathrm{H}_{56}$ & 38.38 & - & - & $2.46 \pm 0.02$ & - & - & - & - & - \\
\hline Nonacosane & $\mathrm{C}_{29} \mathrm{H}_{60}$ & 42.98 & - & - & $6.36 \pm 0.01$ & - & - & - & - & - \\
\hline Octacosane & $\mathrm{C}_{28} \mathrm{H}_{58}$ & 47.26 & - & - & $1.31 \pm 0.02$ & - & - & - & - & - \\
\hline \multicolumn{11}{|l|}{ Aromatic compounds } \\
\hline Isophthalic acid & $\mathrm{C}_{8} \mathrm{H}_{6} \mathrm{O}_{4}$ & 13.9 & $1.19 \pm 0.09$ & - & - & - & - & - & - & - \\
\hline Dibutyl phthalate & $\mathrm{C}_{16} \mathrm{H}_{22} \mathrm{O}_{4}$ & 19.14 & - & $5.49 \pm 0.60$ & $0.69 \pm 0.06$ & $3.91 \pm 0.03$ & $2.51 \pm 0.05$ & $3.91 \pm 0.01$ & $1.05 \pm 0.05$ & $2.48 \pm 0.13$ \\
\hline 1,2-Dihydroxyanthraquinone & $\mathrm{C}_{14} \mathrm{H}_{8} \mathrm{O}_{4}$ & 31.87 & - & - & - & - & - & - & $0.28 \pm 0.04$ & - \\
\hline Bis(2-ethylhexyl) phthalate & $\mathrm{C}_{24} \mathrm{H}_{38} \mathrm{O}_{4}$ & 34.69 & - & - & - & - & - & - & - & $0.79 \pm 0.02$ \\
\hline 4,5-Dihydroxyanthraquinone-2-carboxylic acid & $\mathrm{C}_{15} \mathrm{H}_{8} \mathrm{O}_{6}$ & 41.37 & - & - & $0.47 \pm 0.05$ & - & - & - & - & - \\
\hline \multicolumn{11}{|l|}{ Derivatives from saturated alkanoic acids } \\
\hline 2-Hydroxyheptanoic acid & $\mathrm{C}_{7} \mathrm{H}_{14} \mathrm{O}_{3}$ & 6.41 & $1.23 \pm 0.50$ & $1.40 \pm 0.05$ & - & - & - & - & - & - \\
\hline 3-Phenylpropanoic acid & $\mathrm{C}_{9} \mathrm{H}_{10} \mathrm{O}_{2}$ & 7.12 & - & - & $4.38 \pm 0.09$ & - & - & - & $6.28 \pm 0.33$ & - \\
\hline 3-Hydroxyoctanoic acid & $\mathrm{C}_{8} \mathrm{H}_{16} \mathrm{O}_{3}$ & 7.98 & $0.78 \pm 0.06$ & $0.80 \pm 0.02$ & - & - & - & - & - & - \\
\hline 3-(4-Methoxyphenyl) propionic acid & $\mathrm{C}_{10} \mathrm{H}_{12} \mathrm{O}_{3}$ & 11.49 & - & - & $2.34 \pm 0.11$ & - & - & - & $2.48 \pm 0.13$ & - \\
\hline 2,3-Dihydroxypropyl palmitate & $\mathrm{C}_{19} \mathrm{H}_{38} \mathrm{O}_{4}$ & 36.21 & - & - & - & $0.60 \pm 0.04$ & - & - & - & $0.53 \pm 0.00$ \\
\hline \multicolumn{11}{|l|}{ Derivatives from unsaturated alkanoic acids } \\
\hline Tetradecanoic acid & $\mathrm{C}_{14} \mathrm{H}_{28} \mathrm{O}_{2}$ & 16.07 & - & - & $1.06 \pm 0.02$ & $0.39 \pm 0.00$ & $0.20 \pm 0.01$ & - & $1.56 \pm 0.07$ & $0.42 \pm 0.00$ \\
\hline Pentadecanoic acid & $\mathrm{C}_{15} \mathrm{H}_{30} \mathrm{O}_{2}$ & 18.74 & - & - & - & $0.42 \pm 0.01$ & - & - & - & $0.33 \pm 0.03$ \\
\hline Hexadecanoic acid & $\mathrm{C}_{16} \mathrm{H}_{32} \mathrm{O}_{2}$ & 21.49 & $14.32 \pm 0.45$ & $10.06 \pm 0.13$ & $11.48 \pm 0.12$ & $62.58 \pm 0.36$ & $6.25 \pm 0.08$ & $3.37 \pm 0.03$ & $19.02 \pm 0.02$ & $63.01 \pm 0.07$ \\
\hline Heptadecanoic acid & $\mathrm{C}_{17} \mathrm{H}_{34} \mathrm{O}_{2}$ & 24.22 & - & - & - & $0.85 \pm 0.01$ & $0.32 \pm 0.00$ & $0.17 \pm 0.02$ & - & $0.80 \pm 0.00$ \\
\hline Octadecanoic acid & $\mathrm{C}_{18} \mathrm{H}_{36} \mathrm{O}_{2}$ & 26.93 & $1.90 \pm 0.09$ & $1.49 \pm 0.07$ & $2.93 \pm 0.08$ & $6.09 \pm 0.00$ & $3.11 \pm 0.05$ & $0.70 \pm 0.01$ & $5.14 \pm 0.10$ & $6.91 \pm 0.06$ \\
\hline Eicosanoic acid & $\mathrm{C}_{20} \mathrm{H}_{40} \mathrm{O}_{2}$ & 32.17 & - & - & $0.55 \pm 0.00$ & $1.14 \pm 0.00$ & - & $0.14 \pm 0.01$ & $0.92 \pm 0.03$ & $1.00 \pm 0.01$ \\
\hline \multicolumn{11}{|l|}{ Saturated alkanoic acids } \\
\hline Docosanoic acid & $\mathrm{C}_{22} \mathrm{H}_{44} \mathrm{O}_{2}$ & 37.08 & - & - & $1.19 \pm 0.01$ & $0.84 \pm 0.00$ & - & - & $1.51 \pm 0.05$ & $0.61 \pm 0.01$ \\
\hline Tetracosanoic acid & $\mathrm{C}_{24} \mathrm{H}_{48} \mathrm{O}_{2}$ & 41.71 & - & - & $5.09 \pm 0.03$ & $2.81 \pm 0.01$ & - & - & $3.95 \pm 0.05$ & $2.15 \pm 0.05$ \\
\hline \multicolumn{11}{|l|}{ Saturated diacids } \\
\hline Hexanedioic acid & $\mathrm{C}_{6} \mathrm{H}_{10} \mathrm{O}_{4}$ & 8.49 & $0.91 \pm 0.01$ & $1.79 \pm 0.53$ & - & - & - & $0.19 \pm 0.04$ & - & - \\
\hline Heptanedioic acid & $\mathrm{C}_{7} \mathrm{H}_{12} \mathrm{O}_{4}$ & 10.33 & $1.54 \pm 0.02$ & - & - & - & - & - & - & - \\
\hline Octanedioic acid & $\mathrm{C}_{8} \mathrm{H}_{14} \mathrm{O}_{4}$ & 12.44 & $5.41 \pm 0.36$ & $2.90 \pm 0.34$ & - & $0.96 \pm 0.09$ & $0.18 \pm 0.00$ & $0.21 \pm 0.02$ & - & $0.82 \pm 0.05$ \\
\hline $\begin{array}{l}\text { Nonanedioic acid } \\
\text { Steroids }\end{array}$ & \multicolumn{9}{|c|}{ 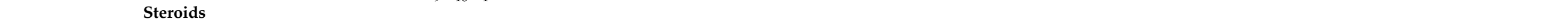 } & $3.97 \pm 0.10$ \\
\hline Campesterol & $\mathrm{C}_{28} \mathrm{H}_{48} \mathrm{O}$ & 50.45 & - & - & - & - & $8.76 \pm 0.07$ & $9.15 \pm 0.20$ & - & - \\
\hline Stigmasterol & $\mathrm{C}_{29} \mathrm{H}_{48} \mathrm{O}$ & 51.14 & - & $1.19 \pm 0.14$ & - & $0.55 \pm 0.08$ & $23.56 \pm 0.19$ & $23.50 \pm 0.11$ & $1.55 \pm 0.02$ & $2.58 \pm 0.08$ \\
\hline$\beta$-Sitosterol & $\mathrm{C}_{29} \mathrm{H}_{50} \mathrm{O}$ & 52.32 & - & - & - & - & $43.76 \pm 0.03$ & $43.43 \pm 0.14$ & - & $1.87 \pm 0.01$ \\
\hline Stigmastanol & $\mathrm{C}_{29} \mathrm{H}_{52}$ & 52.43 & - & - & - & - & $1.30 \pm 0.12$ & $1.04 \pm 0.08$ & - & $1.42 \pm 0.02$ \\
\hline Stigmasta-3,5-dien-7-one & $\mathrm{C}_{29} \mathrm{H}_{46} \mathrm{O}$ & 53.27 & $17.48 \pm 0.35$ & $19.00 \pm 0.29$ & - & - & - & - & - & - \\
\hline
\end{tabular}


Table 3. Cont.

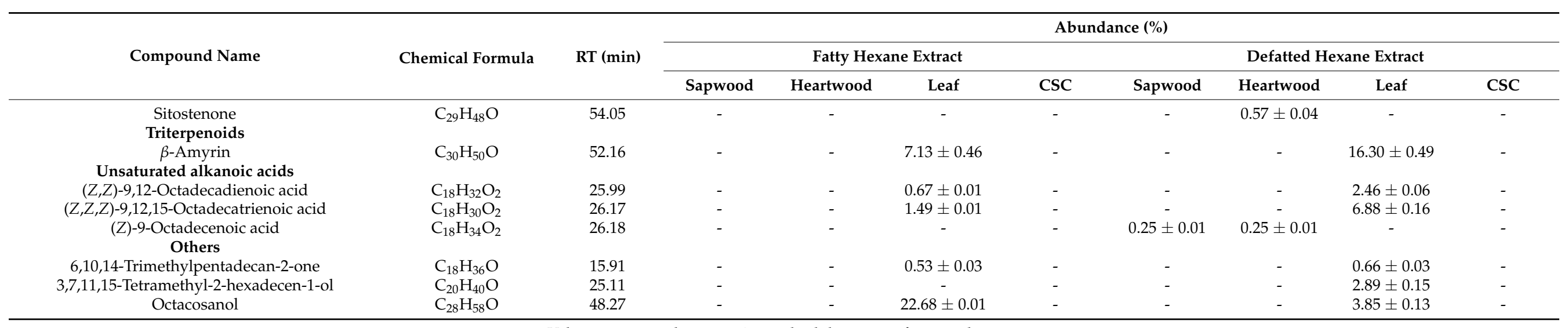

Values represent the mean \pm standard deviation of two replicates.

Table 4. Chemical constituents of the Dichloromethane and methanolic extracts of cell suspension culture (CSC) and plant of Eysenhardtia platycarpa identified by GC-MS.

\begin{tabular}{|c|c|c|c|c|c|c|c|c|c|c|}
\hline \multirow{3}{*}{ Compound Name } & \multirow{3}{*}{ Chemical Formula } & \multirow{3}{*}{ RT (min) } & \multicolumn{8}{|c|}{ Abundance (\%) } \\
\hline & & & \multicolumn{4}{|c|}{ Dichloromethane Extract } & \multicolumn{4}{|c|}{ Methanolic Extract } \\
\hline & & & Sapwood & Heartwood & Leaf & CSC & Sapwood & Heartwood & Leaf & CSC \\
\hline \multicolumn{11}{|l|}{ Aromatic compounds } \\
\hline Vanillin & $\mathrm{C}_{8} \mathrm{H}_{8} \mathrm{O}_{3}$ & 9.09 & $0.53 \pm 0.32$ & - & - & - & - & - & - & - \\
\hline Terephthalic acid & $\mathrm{C}_{8} \mathrm{H}_{6} \mathrm{O}_{4}$ & 14.72 & $0.39 \pm 0.12$ & - & - & - & - & - & - & - \\
\hline Dibutyl phthalate & $\mathrm{C}_{16} \mathrm{H}_{22} \mathrm{O}_{4}$ & 19.14 & $3.05 \pm 0.82$ & $2.97 \pm 0.16$ & - & $0.56 \pm 0.01$ & - & - & - & - \\
\hline 1,2-Dihydroxyanthraquinone & $\mathrm{C}_{14} \mathrm{H}_{8} \mathrm{O}_{4}$ & 31.87 & - & $1.58 \pm 0.01$ & - & $0.43 \pm 0.03$ & - & - & - & - \\
\hline \multicolumn{11}{|l|}{ Derivatives from saturated alkanoic acids } \\
\hline 3-Phenylpropanoic acid & $\mathrm{C}_{9} \mathrm{H}_{10} \mathrm{O}_{2}$ & 7.12 & - & - & $1.02 \pm 0.00$ & - & - & - & - & - \\
\hline 3-(4-Methoxyphenyl) propionic acid & $\mathrm{C}_{10} \mathrm{H}_{12} \mathrm{O}_{3}$ & 11.49 & - & - & $0.65 \pm 0.02$ & - & - & - & - & - \\
\hline \multirow{2}{*}{\multicolumn{11}{|c|}{ Mono and disaccharides }} \\
\hline & & & & & & & & & & \\
\hline Ketohexoses & $\mathrm{C}_{6} \mathrm{H}_{12} \mathrm{O}_{6}$ & * & - & - & - & - & $10.12 \pm 0.10$ & $3.14 \pm 0.08$ & $3.61 \pm 0.02$ & $27.77 \pm 0.03$ \\
\hline Aldohexoses & $\mathrm{C}_{6} \mathrm{H}_{12} \mathrm{O}_{6}$ & * & - & - & - & - & $6.30 \pm 0.32$ & $3.49 \pm 0.24$ & $2.88 \pm 0.07$ & $2.79 \pm 0.00$ \\
\hline Furanoses & $\mathrm{C}_{6} \mathrm{H}_{12} \mathrm{O}_{6}$ & * & - & - & - & - & $4.67 \pm 0.08$ & - & $0.96 \pm 0.02$ & - \\
\hline Disaccharides (glucose with fructose) & $\mathrm{C}_{12} \mathrm{H}_{22} \mathrm{O}_{11}$ & * & - & - & - & - & $28.24 \pm 0.44$ & $3.55 \pm 0.12$ & $1.82 \pm 0.00$ & $40.91 \pm 0.09$ \\
\hline \multicolumn{11}{|l|}{ Polyols } \\
\hline L-threitol & $\mathrm{C}_{4} \mathrm{H}_{10} \mathrm{O}_{4}$ & 8.77 & - & - & - & - & - & - & - & $0.42 \pm 0.00$ \\
\hline Xylitol & $\mathrm{C}_{5} \mathrm{H}_{12} \mathrm{O}_{5}$ & 13.52 & - & - & - & - & $0.40 \pm 0.00$ & $4.23 \pm 0.15$ & - & $2.12 \pm 0.00$ \\
\hline D-pinitol & $\mathrm{C}_{7} \mathrm{H}_{14} \mathrm{O}_{6}$ & 16.38 & - & - & - & - & $37.00 \pm 0.22$ & $15.56 \pm 0.47$ & $74.28 \pm 0.01$ & - \\
\hline
\end{tabular}


Table 4. Cont.

\begin{tabular}{|c|c|c|c|c|c|c|c|c|c|c|}
\hline \multirow{3}{*}{ Compound Name } & \multirow{3}{*}{ Chemical Formula } & \multirow{3}{*}{ RT (min) } & \multicolumn{8}{|c|}{ Abundance (\%) } \\
\hline & & & \multicolumn{4}{|c|}{ Dichloromethane Extract } & \multicolumn{4}{|c|}{ Methanolic Extract } \\
\hline & & & Sapwood & Heartwood & Leaf & $\mathrm{CSC}$ & Sapwood & Heartwood & Leaf & $\mathrm{CSC}$ \\
\hline Sorbitol & $\mathrm{C}_{6} \mathrm{H}_{14} \mathrm{O}_{6}$ & 19.21 & - & - & - & - & - & $2.07 \pm 0.08$ & - & $1.84 \pm 0.00$ \\
\hline Inositol & $\mathrm{C}_{6} \mathrm{H}_{12} \mathrm{O}_{6}$ & * & - & - & - & - & $7.07 \pm 0.05$ & $7.00 \pm 0.22$ & $6.46 \pm 0.02$ & $5.22 \pm 0.01$ \\
\hline \multicolumn{11}{|l|}{ Saturated diacids } \\
\hline Hexanedioic acid & $\mathrm{C}_{6} \mathrm{H}_{10} \mathrm{O}_{4}$ & 8.49 & - & $0.48 \pm 0.02$ & - & - & - & - & - & - \\
\hline Octanedioic acid & $\mathrm{C}_{8} \mathrm{H}_{14} \mathrm{O}_{4}$ & 12.44 & - & $0.74 \pm 0.10$ & - & - & - & - & - & - \\
\hline Nonanedioic acid & $\mathrm{C}_{9} \mathrm{H}_{16} \mathrm{O}_{4}$ & 14.87 & $1.94 \pm 0.09$ & $4.53 \pm 0.73$ & $0.51 \pm 0.01$ & $0.63 \pm 0.01$ & $0.44 \pm 0.09$ & $1.17 \pm 0.49$ & - & - \\
\hline \multicolumn{11}{|l|}{ Saturated alkanoic acids } \\
\hline Tetradecanoic acid & $\mathrm{C}_{14} \mathrm{H}_{28} \mathrm{O}_{2}$ & 16.07 & - & - & $0.36 \pm 0.01$ & - & - & - & - & - \\
\hline Hexadecanoic acid & $\mathrm{C}_{16} \mathrm{H}_{32} \mathrm{O}_{2}$ & 21.49 & $27.42 \pm 0.26$ & $22.40 \pm 0.26$ & $5.20 \pm 0.00$ & $35.02 \pm 0.44$ & - & - & $2.06 \pm 0.00$ & $2.05 \pm 0.00$ \\
\hline Heptadecanoic acid & $\mathrm{C}_{17} \mathrm{H}_{34} \mathrm{O}_{2}$ & 24.22 & $0.77 \pm 0.13$ & $0.87 \pm 0.02$ & - & $0.36 \pm 0.00$ & - & - & - & - \\
\hline Octadecanoic acid & $\mathrm{C}_{18} \mathrm{H}_{36} \mathrm{O}_{2}$ & 26.93 & $3.37 \pm 0.21$ & $3.21 \pm 0.04$ & $1.44 \pm 0.00$ & $2.75 \pm 0.22$ & - & - & $1.88 \pm 0.01$ & $1.16 \pm 0.03$ \\
\hline Eicosanoic acid & $\mathrm{C}_{20} \mathrm{H}_{40} \mathrm{O}_{2}$ & 32.17 & $0.58 \pm 0.04$ & - & - & $0.42 \pm 0.00$ & - & - & - & - \\
\hline Docosanoic acid & $\mathrm{C}_{22} \mathrm{H}_{44} \mathrm{O}_{2}$ & 37.08 & $0.54 \pm 0.01$ & - & - & $0.29 \pm 0.01$ & - & - & - & - \\
\hline Tetracosanoic acid & $\mathrm{C}_{24} \mathrm{H}_{48} \mathrm{O}_{2}$ & 41.71 & $1.25 \pm 0.10$ & $0.90 \pm 0.00$ & $0.96 \pm 0.03$ & $1.27 \pm 0.01$ & - & - & - & - \\
\hline \multicolumn{11}{|l|}{ Sesquiterpenoids } \\
\hline$\beta$-Selinene & $\mathrm{C}_{15} \mathrm{H}_{24}$ & 8.31 & - & - & $0.46 \pm 0.01$ & - & - & - & - & - \\
\hline$\gamma$-Muurolene & $\mathrm{C}_{15} \mathrm{H}_{24}$ & 8.74 & - & - & $0.32 \pm 0.01$ & - & - & - & - & - \\
\hline$\beta$-Cadinene & $\mathrm{C}_{15} \mathrm{H}_{24}$ & 11.2 & - & - & $0.77 \pm 0.03$ & - & - & - & - & - \\
\hline Campesterol & $\mathrm{C}_{28} \mathrm{H}_{48} \mathrm{O}$ & 50.45 & $1.61 \pm 0.11$ & $1.76 \pm 0.11$ & - & $0.82 \pm 0.11$ & - & - & - & - \\
\hline Stigmasterol & $\mathrm{C}_{29} \mathrm{H}_{48} \mathrm{O}$ & 51.14 & $4.06 \pm 0.41$ & $3.96 \pm 0.16$ & - & $6.72 \pm 0.05$ & - & - & - & - \\
\hline$\beta$-Sitosterol & $\mathrm{C}_{29} \mathrm{H}_{50} \mathrm{O}$ & 52.32 & $8.19 \pm 0.63$ & $7.50 \pm 0.55$ & - & $6.00 \pm 0.07$ & - & - & - & - \\
\hline \multicolumn{11}{|l|}{ Unsaturated alkanoic acids } \\
\hline$(Z, Z)-9,12-$-Octadecadienoic acid & $\mathrm{C}_{18} \mathrm{H}_{32} \mathrm{O}_{2}$ & 25.99 & $3.99 \pm 0.06$ & $5.20 \pm 0.05$ & $0.72 \pm 0.03$ & $13.63 \pm 0.16$ & - & - & - & - \\
\hline$(Z, Z, Z)-9,12,15$-Octadecatrienoic acid, & $\mathrm{C}_{18} \mathrm{H}_{30} \mathrm{O}_{2}$ & 26.17 & - & - & $2.29 \pm 0.00$ & $3.79 \pm 0.06$ & - & - & - & - \\
\hline (Z)-9-Octadecenoic acid & $\mathrm{C}_{18} \mathrm{H}_{34} \mathrm{O}_{2}$ & 26.18 & $1.62 \pm 0.04$ & $2.49 \pm 0.05$ & - & $0.82 \pm 0.01$ & - & - & - & - \\
\hline \multicolumn{11}{|l|}{ Others } \\
\hline Malic acid & $\mathrm{C}_{4} \mathrm{H}_{6} \mathrm{O}_{5}$ & 8.3 & - & - & - & - & $1.95 \pm 0.01$ & - & - & - \\
\hline L-Threonic acid & $\mathrm{C}_{4} \mathrm{H}_{8} \mathrm{O}_{5}$ & 9.79 & - & - & - & - & - & - & $0.42 \pm 0.00$ & - \\
\hline Galactaric acid & $\mathrm{C}_{6} \mathrm{H}_{10} \mathrm{O}_{8}$ & 20.16 & - & - & - & - & - & $1.68 \pm 0.06$ & - & - \\
\hline 3,7,11,15-Tetramethyl-2-hexadecen-1-ol & $\mathrm{C}_{20} \mathrm{H}_{40} \mathrm{O}$ & 25.11 & - & - & $1.06 \pm 0.00$ & - & - & - & - & - \\
\hline
\end{tabular}

Values represent the mean \pm standard deviation of two replicates. * Several stereoisomers were found at different retention times. 
Chromatograms with the main compounds identified by GC-MS are in Figures S1-S16 and their mass spectra are in Figures S17-S29.

All hexane extracts of sapwood, heartwood, leaf, and CSC were composed mostly of saturated alkanoic acids (Saa), saturated diacids (Sd), and steroids (Ste), while only the leaves had triterpenes (Tri) (Figure 4A,B). The fatty and defatted hexane extracts of CSC stood out for producing saturated alkanoic acids (75\%), mainly hexadecanoic acid $(63 \%)$ (Table 3; Figures S4 and S8).
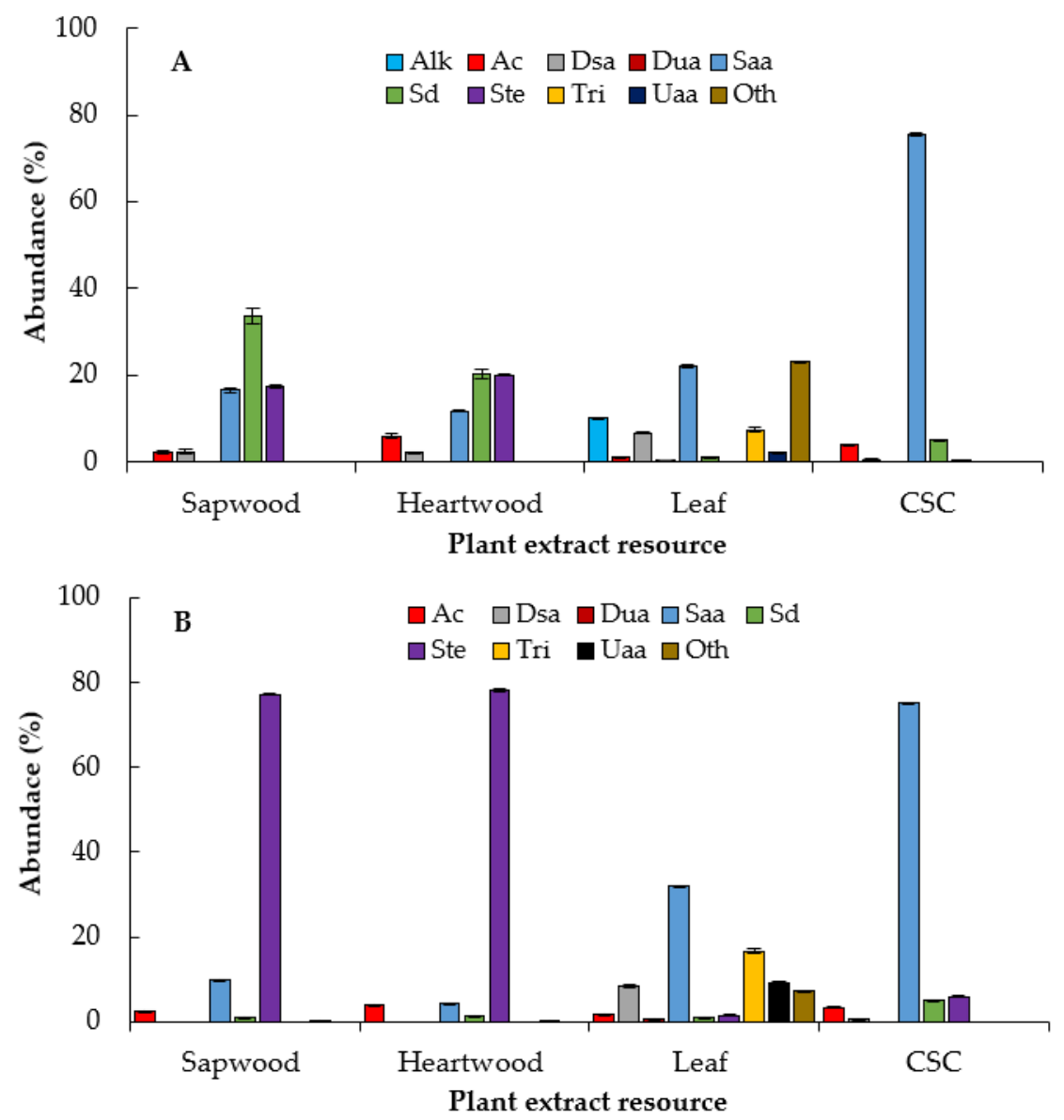

Figure 4. Chemical groups summarized by plant extracts resource of E. platycarpa. (A) Fatty hexane extract; (B) defatted hexane extract. Alk: alkanes; Ac: aromatic compounds; Dsa: derivatives from saturated alkanoic acids; Dua: derivatives from unsaturated alkanoic acids; Saa: saturated alkanoic acids; Sd: saturated diacids; Ste: steroids; Tri: triterpenoids; Uaa: unsaturated alkanoic acids; Oth: others. CSC: Cell suspension cultures. Values represent the mean \pm standard deviation of two replicates.

The largest amount of saturated diacids (nonanedioic acid) were found in the fatty hexane extract of sapwood (26.9\%) and heartwood (15.3\%) (Figures S1 and S2). On the other hand, high amounts of steroids were found in defatted hexane extracts of sapwood (77.2\%) and heartwood (78.1\%), mainly composed of $\beta$-sitosterol ( 43\%) and stigmasterol $(\sim 23 \%)$ (Table 3; Figures S5 and S6). It is possible that these compounds are found as natural derivatives in leaf extracts because only stigmasterol was found at a low abundance (1.55\%), but the peaks at the retention times 52.16-53.05 showed a complex fragmentation pattern that was not possible to characterize (Figures S3 and S7). Stigmasta-3,5-dien-7-one was also found in the fatty hexane extracts of sapwood and heartwood in amounts of $17.48 \%$ and $19.0 \%$, respectively. Only the fatty and defatted hexane extracts from the leaves contained $\beta$-amyrin in amounts of $7.13 \%$ and $16.30 \%$, respectively (Table 3; Figures S3 and S7).

In several studies, it has been shown that hexane extracts are mainly composed of fatty acids with hydrocarbons and traces of terpenes-for example, in the hexane extracts 
of Anisopus mannii leaves, $73.8 \%$ of compounds were identified, highlighting hexadecanoic acid $(34 \%)$, hexadecyl oxirane (11\%), and $(Z, Z, Z)-9,12,15$-octadecatrienoic acid (9.6\%), which showed antimicrobial activity [38]. The hexane extracts of the heartwood of other Fabaceae such as Robinia pseudoacacia L. are also rich in hexadecanoic acid, trimethylsilyl ester (13.39\%), (Z,Z)-9,12-octadecadienoic acid (10.10\%), tetradecane (6.88\%), bis-(2-ethylhexyl) phthalate $(6.21 \%)$, and hexadecane $(6.15 \%)$ [39].

In general, dichloromethane extracts of E. platycarpa were dominated mostly by saturated alkanoic acids and steroids, and unsaturated alkanoic acids (Uaa), and lesser amounts of aromatic compounds (Ac), saturated diacids (Sd), and sesquiterpenoids (Ses) (Figure 5A).
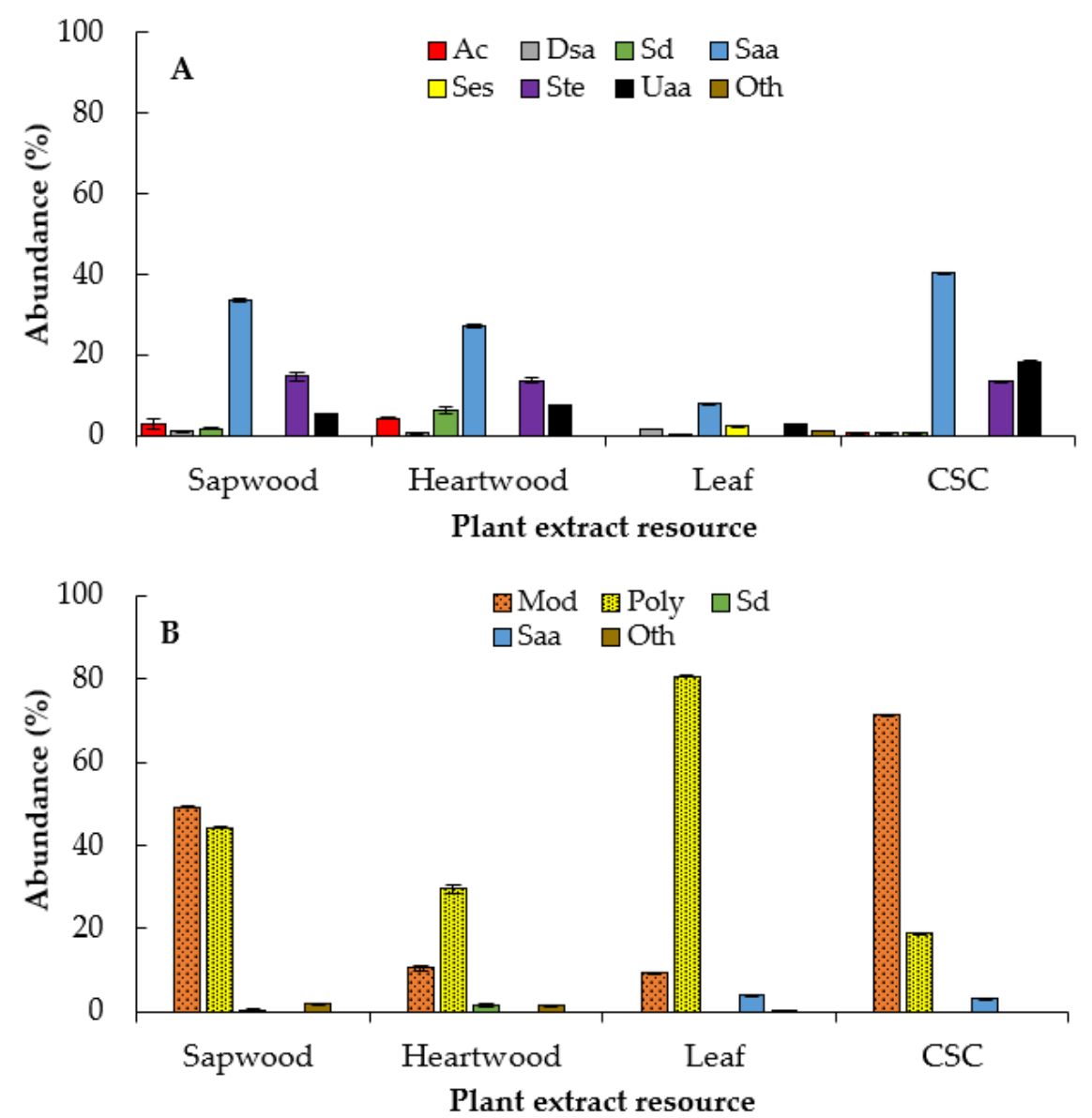

Figure 5. Chemical groups of E. platycarpa extracts, summarized by plant extract source. (A) Dichloro methane extract; (B) methanolic extract. Ac: aromatic compounds; Dsa: derivatives from saturated alkanoic acids; Mod: mono and disaccharides; Poly: polyols; Sd: saturated diacids; Saa: saturated alkanoic acids; Ses: sesquiterpenoids; Ste: steroids; Uaa: unsaturated alkanoic acids; Oth: others. CSC: Cell suspension cultures. Values represent the mean \pm standard deviation of two replicates.

Dichloromethane extract of CSC showed a greater amount of saturated alkanoic acids (35.3\% of hexadecanoic acid) and unsaturated alkanoic acids (13.8\% of (Z,Z)-9,12octadecadienoic acid) (Table 4 and Figure S12). The dichloromethane extracts of sapwood, heartwood, and CSC had similar amounts of steroids, from $13.5 \%$ to $14.7 \%$. Only the leaves exhibited sesquiterpenoids ( $\beta$-selinene, $\gamma$-muurolene, $\beta$-cadinene, 11-hydroxy- $4 \beta \mathrm{H}, 5 \alpha$ eremophil-1(10)-ene and trans, trans-farnesol) in amounts $<1 \%$. In contrast, all methanolic extracts exhibited polyol-type compounds (Figure 5B), mainly D-pinitol in the leaf extracts, with $74.3 \%$ (Table 4 and Figure S13). The rest of the compounds were mostly mono- and disaccharides, mainly in the CSC extracts

In the dichloromethane fractions of sapwood and heartwood extracts from Quercus faginea, saturated alkanoic acids have been found as the main constituents $(15.7 \%$ and 
$25.8 \%$ in sapwood and heartwood, respectively), with hexadecanoic acid being the main compound (35.5\% and $41.1 \%$ ). In addition, sterols have been found between $10.2 \%$ to $13.0 \%$, mainly $\beta$-sitosterol at $6.6 \%$ to $12.1 \%$ [40]. In a study, we found that in extracts with a mixture of chloroform and methanol (1:1) of Cnidoscolus chayamansa cell suspension cultures, lupeol acetate was obtained (38.1 mg/g extract), and the extracts had antibacterial activity [41]. Nonanedioic acid, $\beta$-sitosterol, stigmasterol, stigmasta-3,5-dien-7-one, $\beta$-amyrin, $(Z, Z)$ 9,12-octadecadienoic acid, D-pinitol, hexadecanoic acid, and other compounds have been reported for Eysenhardtia genus and some Fabaceae species [10,11,42]; however, in this work, the phytochemical screening by GC-MS analysis of plants and cell suspension culture extracts from E. platycarpa is reported for the first time.

\subsection{Antifungal Activity}

As an assay of biological activity, we evaluate the antifungal potential of hexanic (fatty and defatted), dichloromethane, and methanolic extracts from sapwood, heartwood, leaves, and cell suspension cultures on phytopathogenic fungi available in our laboratory (Rhizoctonia solani and Sclerotium cepivorum). These fungi have a broad host range and cause significant losses in terms of the yield and quality of many crop species. In a previous study, we reported the antifungal activity of extracts of Eysenhardtia polystachya [13], a species close to E. platycarpa. In the current work, it was found that the antifungal activity of E. platycarpa extracts was statistically significant $(p=0.05)$ on the mycelial growth of $R$. solani and S. cepivorum (Figures 6 and 7).

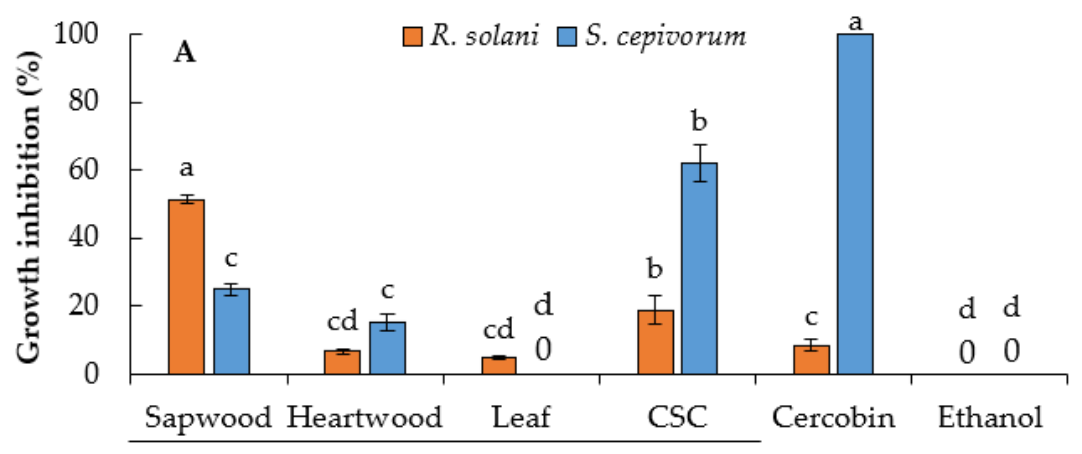

Plant extract resoure

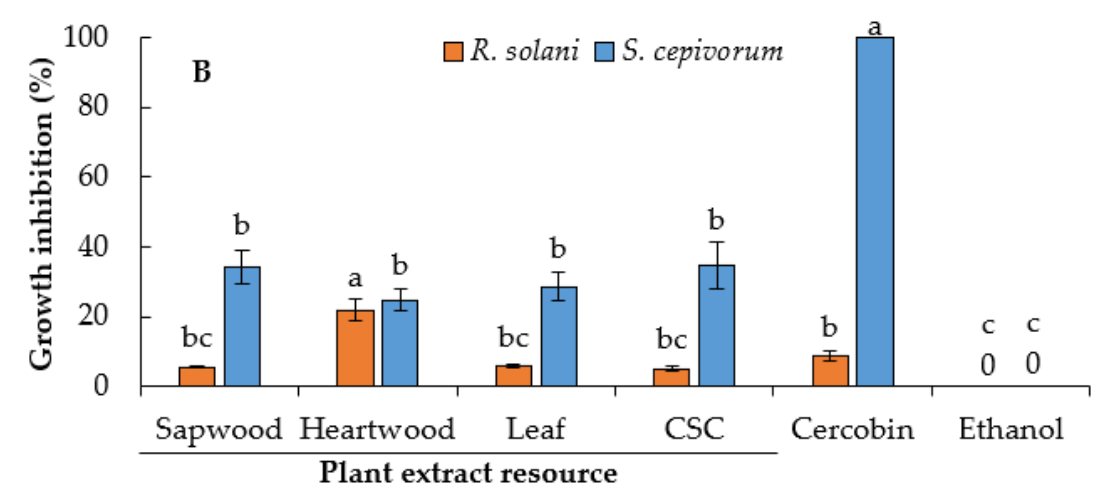

Figure 6. Effect of different source extracts of Eysenhardtia platycarpa on inhibition of mycelial growth of Rhizoctonia solani and Sclerotium cepivorum after $72 \mathrm{~h}$ of culture. (A) Fatty hexane extract; (B) defatted hexane extract. CSC: Cell suspension culture. Values represent mean \pm standard deviation of three replicates. Bars followed by the same letter between the same fungus are not significantly different $(p=0.05)$ using Tukey's multiple range test. 

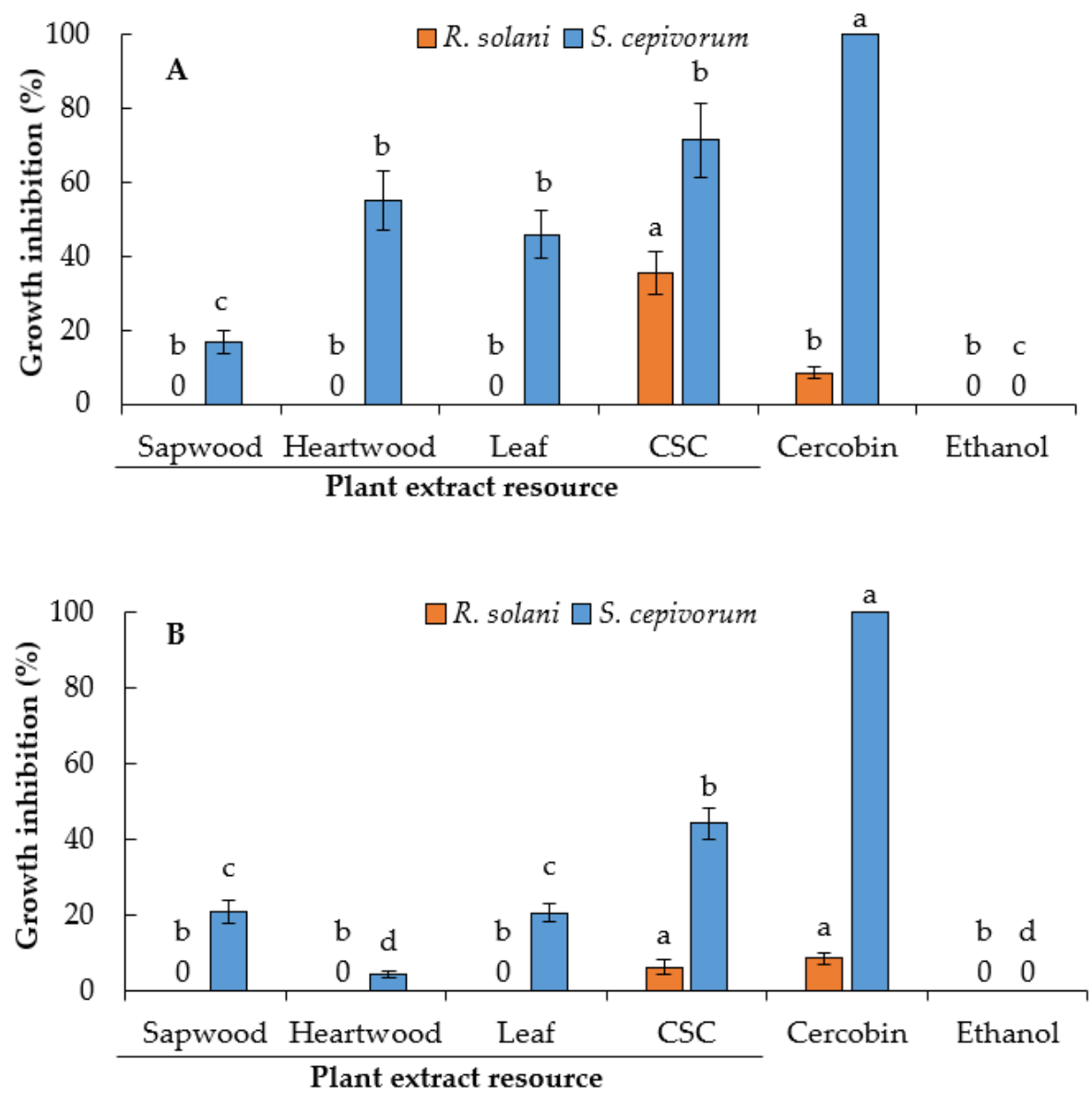

Figure 7. Effect of different source extracts of Eysenhardtia platycarpa on the inhibition of mycelial growth of Rhizoctonia solani and Sclerotium cepivorum at $72 \mathrm{~h}$ of culture: (A) Dichloromethane extract; (B) methanolic extract. CSC: Cell suspension culture. Values represent mean \pm standard deviation of three replicates. Bars followed by the same letter between the same fungus are not significantly different $(p=0.05)$ using Tukey's multiple range test.

Of the 16 extracts, only the fatty hexane and dichloromethane extracts showed better inhibition of both fungi species. The fatty hexane extract of sapwood had the maximum inhibition of mycelial growth for $R$. solani (51.4\%), while the fatty hexane extract of cell suspension culture (CSC) showed $62.0 \%$ inhibition for S. cepivorum (Figure 6A). The other fatty hexane extracts had inhibition values lower than $25.0 \%$. In the case of defatted hexane extracts, the inhibition percentages for $R$. solani were low (less than $22 \%$ ), while the values for S. cepivorum ranged from $22 \%$ to $35 \%$ (Figure $6 \mathrm{~B}$ ). The fungicide Cercobin did not affect the growth of $R$. solani but was efficient at inhibiting the growth of $S$. cepivorum. In a previous study, we reported the antifungal activity of E. polystachya extracts, in which the defatted hexane extract of CSC showed $66.0 \%$ inhibition for $R$. solani and was also higher than Cercobin, while the fatty hexane extracts had low inhibition [13].

Regarding the dichloromethane extracts, only the cell suspension cultures extract showed moderate inhibition of $R$. solani (36.0\%) compared with Cercobin (9.0\%) (Figure 7A).

In contrast, the dichloromethane extract from the cell suspension culture showed the most effective inhibition against S. cepivorum (71.5\%), followed by heartwood (55.2\%) and leaf extracts (45.9\%) (Figure 7A). In a study carried out on E. polystachya, we reported that dichloromethane extracts of sapwood and heartwood also inhibited the growth of S. cepivorum by $73.0 \%$ and $80.0 \%$, respectively [13].

On the other hand, the methanolic extracts from E. platycarpa showed low inhibition compared with Cercobin. However, CSC extracts showed the maximum percentage of inhibition for S. cepivorum, 44.2\% (Figure 7B). 
Many plants have been reported to inhibit the in vitro growth of phytopathogenic fungi, which promise to be better than commercial fungicides [43,44]. It is possible that the growth inhibition of $R$. solani and S. cepivorum with the fatty hexane extract of the sapwood and CSC (Figure 6A) is due to a synergism between saturated fatty acids, saturated diacids, and steroids, since these are more abundant in this extract (Figure $4 \mathrm{~A}$ and Table 3 ). In fact, the defatted hexanic extracts also contain saturated fatty acids and a high amount of steroids, but scarce saturated diacids compared with the fatty hexane extract. Therefore, there was also a decrease in the inhibition of fungal growth (Figure 6B).

Several free fatty acids (lauric acid, myristic acid, palmitic acid, oleic acid, and linoleic acid) are known to have an inhibitory effect on fungal germination, mycelial growth, and sporulation $[45,46]$. The possible mechanisms of antifungal activity have been studied previously and focused on fungal membrane disruption, causing an increase in membrane fluidity, causing leakage of the intracellular components and cell death [47] or interfering fungal sphingolipid biosynthesis [48]. In addition, they may influence the inhibition of protein and enzyme synthesis related to fatty acid metabolism [49]. On the other hand, the synergism of the aromatic compounds, along with the high amounts of phytosterols (stigmasterol and $\beta$-sitosterol), hexadecanoic acid, and unsaturated fatty acids $(Z, Z)-9,12$ octadecadienoic acid) found in CSC dichloromethane extract of E. platycarpa (Table 4) may have increased the inhibition for S. cepivorum. On the other hand, the CSC dichloromethane extract was the only one that significantly inhibited the growth for $R$. solani, perhaps due to the high amount of saturated and unsaturated alkanoic acids and steroids compared to the other dichloromethane extracts from intact plants (Figures 5A and 7A).

The hexadecanoic acid (palmitic acid) from many plant species has been reported against phytopathogenic fungi, such as Aspergillus niger, Botrytis cinerea, Colletotrichum lagenarium, Emericella nidulans, Fusarium oxysporum, and Alternaria solani $[45,49,50]$. Phytosterols, e.g., stigmasterol and sitosterol, have also been reported to be effective against phytopathogenic fungi [51,52]. A mixture of stigmasterol and $\beta$-sitosterol isolated from the pericarp of Areca catechu markedly inhibited the spore germination, mycelial growth, and germ-tube elongation of Colletotrichum gloeosporioides [53]. Studies reported in other species of the Fabaceae family (Tephrosia apollinea, Dahlstedtia glaziovii, and Deguelia duckeana), have shown that dichloromethane extracts can inhibit fungal growth because they contain high amounts of flavonoids and prenylated phenolic compounds $[26,54,55]$. Therefore, some plant extracts may be a source of antifungal compounds since they have had to develop compounds to resist infections by fungi present in their environment [56].

\section{Materials and Methods}

\subsection{Collection of Plant Material}

The leaves, seeds, and the trunk from wild Eysenhardtia platycarpa plants were collected in November 2015, in San Luciano (Jocotepec, Estado de Jalisco, Mexico), located at $20^{\circ} 19^{\prime} 10.23^{\prime \prime} \mathrm{N}$ and $103^{\circ} 24^{\prime} 12^{\prime \prime} \mathrm{W}$ at an elevation of $1950 \mathrm{~m}$ a.s.l. A sample of the plant was used for identification, registered, and deposited in the Luz María Villarreal de Puga Herbarium, Instituto de Botanica, Universidad de Guadalajara (IBUG) with the voucher number 28112017.

\subsection{Obtaining Plantlets and Incubation Conditions}

All in vitro culture experiments were conducted at the Laboratorio de Cultivo In vitro de Plantas of the Departamento de Madera, Celulosa y Papel, of the Universidad de Guadalajara, México. First, uncoated seeds of E. platycarpa were washed with a soap solution for $10 \mathrm{~min}$, followed by disinfection with $70 \%(v / v)$ ethanol for $30 \mathrm{~s}$. Then, the seeds were disinfected with $1.2 \%(v / v)$ sodium hypochlorite solution (Cloralex ${ }^{\circledR}$, Industrias Alen, S.A. de C.V, Moterrey, Nuevo León, México) for $15 \mathrm{~min}$ along with four drops of Tween $20^{\circledR}$ per $100 \mathrm{~mL}$ of disinfectant solution. After disinfection, the seeds were rinsed three times with sterile distilled water in a horizontal laminar flow cabinet (CFLH-90E, Novatech, Guadalajara, Jal. Mexico). 
The disinfected seeds were sown in MS culture medium [57] supplemented with $3 \%$ sucrose $(w / v)$ (Sigma-Aldrich, St. Louis, MO, USA); only half of the macronutrients were used. The culture medium was adjusted to $\mathrm{pH} 5.8$ and then gelled with $2 \mathrm{~g} / \mathrm{L}$ of Phytagel ${ }^{\circledR}$ (Sigma-Aldrich). The culture medium was transferred to Gerber flasks of $100 \mathrm{~mL}$ capacity and sterilized in a manual autoclave (CV300-A, AESA, Tecamac, Estado de México, México) at $121^{\circ} \mathrm{C}, 15 \mathrm{psi}$, for $18 \mathrm{~min}$. Four disinfected seeds were placed in Gerber-type jars containing $25 \mathrm{~mL}$ of MS medium.

All cultures were incubated at $25 \pm 2{ }^{\circ} \mathrm{C}$ under a 16-h photoperiod of white fluorescent light with a light intensity of $60 \mu \mathrm{mol} / \mathrm{m}^{2} / \mathrm{s}$. Eight days after germination, the plantlets were transferred to a flask with a $1 \mathrm{~L}$ capacity, containing $80 \mathrm{~mL}$ of MS culture medium. Plantlets were used for subsequent experiments on callus induction.

\subsection{Establishment of Callus Cultures}

To induce calluses, 30-day-old plantlets grown in in vitro conditions were used as a source of explants. Internodal segments of approximately $1 \mathrm{~cm}$ in length were sown in Gerber flasks containing $25 \mathrm{~mL}$ of semisolid MS culture medium and plant growth regulators (PGRs). The PGRs consisted of two auxins, 2,4-dichlorophenoxyacetic acid $(2,4-\mathrm{D})$ or naphthaleneacetic acid (NAA), each combined with kinetin (KIN) as a cytokinin; all PGRs were used at $0.0,0.1,1.0$, and $2.0 \mathrm{mg} / \mathrm{L}$. Each treatment consisted of four flasks with four explants per flask $(n=16)$, and the experiment was repeated twice. Cultures were maintained under the same incubation conditions as in seed germination and subcultured every four weeks with fresh culture medium.

\subsection{Cell Suspension Cultures}

\subsubsection{Growth Kinetics}

The calluses that seemed more friable and showed better growth characteristics were used to initiate the establishment of the cell suspension cultures (CSC), using MS liquid medium with the same PGRs that induced the callus. Erlenmeyer flasks ( $125 \mathrm{~mL}$ capacity) containing $25 \mathrm{~mL}$ of culture medium were inoculated with $3 \mathrm{~g}$ of callus (FW). Cultures were incubated in an orbital shaker (PRENDO AGO-6040; Puebla, Pue. Mexico) at $110 \mathrm{rpm}$ under a photoperiod ( $16 \mathrm{~h} \mathrm{light} / 8 \mathrm{~h}$ dark) of white fluorescent light with a light intensity of $60 \mu \mathrm{mol} / \mathrm{m}^{2} / \mathrm{s}$ and $25 \pm 2{ }^{\circ} \mathrm{C}$. When an increase of biomass was shown in the flasks, cells were harvested and filtered with 200- $\mu$ m nylon mesh filters (Whatman No. 1) to remove excess culture medium. Then cells were subcultured every two weeks for six months in several flasks to increase the biomass, and no changes were observed in the culture. Kinetic growth was carried out in an Erlenmeyer flask ( $125 \mathrm{~mL}$ capacity) containing $25 \mathrm{~mL}$ of MS liquid medium and $1.5 \mathrm{~g}$ of fresh cells. The biomass contained in the three flasks was harvested every two days, filtered, and washed with distilled water to remove the excess culture medium. The biomass was dried in an oven at $50{ }^{\circ} \mathrm{C}$ until a constant weight. The experiment was repeated twice, and the biomass dry weight (DW) data were used to plot the growth curve. The value of the specific growth rate $(\mu)$ was calculated by plotting the natural logarithm of biomass versus time, between days 2 and 8 of growth culture (exponential phase). The slope of this linear part of the kinetics was defined as $\mu$ and is given in $1 /$ unit of time $\left(\right.$ day $\left.^{-1}\right)$. The generation (doubling) time $(t d)$ was calculated from the $\mu$ value and expressed as $t d$ (day) $=\ln (2) / \mu$, and the growth index was calculated considering the maximal biomass obtained with a reduction of the inoculum and divided by the inoculum. Biomass produced according to the sucrose content was determined based on the theoretical value $(\mathrm{Y}=\mathrm{g}$ of maximum biomass/g of sucrose added to culture medium) reported for plants $[20,23,58]$. The modified Gompertz model is well known and widely used in many aspects of biology and has been frequently used to describe the plant growth [21,22]. The lag phase and other growth parameters were estimated with the modified Gompertz model [59] using Equation (1):

$$
\mathbf{X}_{\mathbf{t}}=X_{\max } \cdot \exp \left\{-\exp \left[\frac{\mu \mathrm{e}}{X_{\max }}(\lambda-t)+1\right]\right\}
$$


where $\mathbf{X}_{\mathbf{t}}(\mathrm{g} / \mathrm{L})$ is the biomass at any time $t$ (days), $\mu\left(\right.$ day $\left.^{-1}\right)$ is the specific growth rate, $X_{\max }(\mathrm{g} / \mathrm{L})$ is the maximum cell growth achieved during the stationary phase, $\lambda$ is the lag time (days), and e $=2.7182$. The nonlinear regression was performed using the Kaleida Graph (4.0 Synergy Software, Reading, PA, USA) to predict the kinetic parameters.

After determining the growth kinetics, cells were subcultured every 12 days for three months. The accumulated biomass was washed with distilled water, dried at $40{ }^{\circ} \mathrm{C}$, and stored frozen until subsequent experiments.

\subsubsection{Sucrose Consumption Determination}

The culture medium filtered from the CSC of the growth kinetics was used to determine the total sugars. For each sampling, three flasks were harvested, and an aliquot of $5 \mathrm{~mL}$ was taken from each flask $(n=3)$ and stored frozen until analysis by the phenolsulfuric method [60]. An aliquot of $250 \mu \mathrm{L}$ of culture medium was diluted in distilled water (1:400); then, a $500-\mu \mathrm{L}$ aliquot was taken and $500 \mu \mathrm{L}$ of phenol $(5 \%)$ was added; subsequently, $2.5 \mathrm{~mL}$ of concentrated sulfuric acid were added. The sample was vigorously mixed for $3 \mathrm{~s}$ and allowed to react at room temperature for $30 \mathrm{~min}$. Samples were read in a spectrophotometer at $490 \mathrm{~nm}$, using distilled water as a blank. To carry out the calibration curve, sucrose was used as a standard at concentrations of 1-40 g/L.

\subsubsection{Determination of the Total Phenolics and Flavonoids Content}

Biomass samples of CSC from growth kinetics and wild plant leaf were used to determine the total phenolics and flavonoids content. Samples of $50 \mathrm{mg}$ of CSC or leaves were refluxed using $20 \mathrm{~mL}$ methanol (in water bath) at $65^{\circ} \mathrm{C}$ for $20 \mathrm{~min}$, and three extraction cycles were done for each sample. The extracts of the same samples were mixed, filtered, brought up to $20 \mathrm{~mL}$, transferred to amber flasks, and stored frozen until analysis.

The total phenolics content (TPH) was quantified by the Folin-Ciocalteu (FC) method [61]. An aliquot of $500 \mu \mathrm{L}$ methanolic extract was mixed with $125 \mu \mathrm{L}$ of FC and then $125 \mu \mathrm{L} \mathrm{Na}_{2} \mathrm{CO}_{3}(20 \% \mathrm{w} / v)$ was added. The mixture was supplemented with distillated water up to a $2 \mathrm{~mL}$ total volume. The reaction was maintained at room temperature for $60 \mathrm{~min}$ in the dark. TPH was calculated based on the calibration curve of gallic acid at concentrations of 0 to $50 \mathrm{mg} / \mathrm{L}$. Samples of methanolic extracts from leaves and CSC were analyzed in a Varian Cary ${ }^{\circledR} 50 \mathrm{UV}$-Vis spectrophotometer (Agilent Technologies, Inc., Santa Clara, CA, USA) at $765 \mathrm{~nm}$. The results were expressed in terms of gallic acid equivalents (GAE) in $\mathrm{mg} / \mathrm{g}$ of dry biomass (DW).

The total flavonoids content (TFL) was quantified with the aluminum chloride colorimetric method [62]. An aliquot of methanolic extracts $(240 \mu \mathrm{L})$ was mixed with $1.50 \mathrm{~mL}$ of distilled water, then $90 \mu \mathrm{L} \mathrm{NaNO}_{2}(5 \%)$ was added and allowed to react for $6 \mathrm{~min}$ in the dark. After the reaction, $180 \mu \mathrm{L} \mathrm{AlCl}_{3}(10 \%)$ was added to the mixture, which was stirred vigorously. After $5 \mathrm{~min}, 600 \mu \mathrm{L} \mathrm{NaOH}(1 \mathrm{M})$ was added and brought up to a $3 \mathrm{~mL}$ final volume with distilled water. Samples were analyzed in a Varian Cary ${ }^{\circledR} 50 \mathrm{UV}$-Vis spectrophotometer at $510 \mathrm{~nm}$. The calibration curve was performed with quercetin as the standard, using concentrations of 100 to $1600 \mu \mathrm{g} / \mathrm{mL}$. The results were expressed in terms of quercetin equivalents $(\mathrm{QE})$ in $\mu \mathrm{g} / \mathrm{g}$ of dry biomass (DW).

\subsection{Phytochemical Analysis of Cell Suspension Cultures and Intact Plants \\ 3.5.1. Extraction and Sample Preparation}

The extraction process was carried out according to a previously reported methodology to obtain extracts or bioactive compounds from E. platycarpa or E. polystachya $[12,13,63]$. The biomass of the 12-day cell suspension cultures and samples from the intact plant (sapwood, heartwood, and leaves) were ground to a fine powder and dried in an oven at $50{ }^{\circ} \mathrm{C}$. Separately, dried samples of sapwood (300 g), heartwood $(269 \mathrm{~g})$, leaves (238 g), and CSC (9.91 g) were extracted (four each) by maceration at room temperature with hexane, dichloromethane, and methanol for $72 \mathrm{~h}$. After the extraction of each solvent, the samples were dried before adding the next solvent. The extracts were filtered and 
concentrated using a rotavapor BÜCHI EL-131 (BÜCHI Labortechnik AG, Flawil, Switzerland) and dried in an oven at $40{ }^{\circ} \mathrm{C}$ to remove traces of the solvent. Each hexane extract was extracted with methanol to obtain two extracts (a defatted hexane extract and a fatty hexane extract for each sample). The yield percentages were obtained with Equation (2):

$$
\text { Yield }(\%)=\left(\frac{d e}{d b}\right) \times 100
$$

where $d e$ is the dry weight of the extract and $d b$ is the dry weight of the biomass used for extraction.

A total of 16 dry extracts were obtained and derivatized with BSTFA to identify as many compounds as possible by GC-MS analysis. The derivatization was carried out according to the methodology previously reported for extracts from Quercus faginea and E. polystachya [40,63]. Briefly, $2 \mathrm{mg}$ of extracts were dissolved in $100 \mu \mathrm{L}$ of pyridine and derivatized by adding $100 \mu \mathrm{L}$ of bis(trimethylsilyl)trifluoroacetamide (BSTFA). The reaction mixture was heated to $50{ }^{\circ} \mathrm{C}$ and stirred for $30 \mathrm{~min}$ on a heating plate.

\subsubsection{Analysis of Extracts by Gas Chromatography-Mass Spectrometry (GC-MS)}

The derivatized extracts $(1 \mu \mathrm{L})$ were immediately injected into an Agilent 6890 instrument coupled to an Agilent Technologies 5973N Network Mass Selective Detector (GC-MS) using electron impact as the ion source at $70 \mathrm{eV}$ in a mass range of 20-600 DA (Agilent Technologies, Inc., Santa Clara, CA, USA). The capillary column was a HP-5MS $(30 \mathrm{~m} \times 0.25 \mathrm{~mm}, 0.25 \mu \mathrm{m}$ film thickness; Agilent Technologies, Inc.). The oven temperature was initially set to $100{ }^{\circ} \mathrm{C}$ (for $1 \mathrm{~min}$ ) and rose at a rate of $10^{\circ} \mathrm{C} / \mathrm{min}$ up to $150{ }^{\circ} \mathrm{C}$, then at a rate of $3{ }^{\circ} \mathrm{C} / \mathrm{min}$ up to $300^{\circ} \mathrm{C}$ (for $4 \mathrm{~min}$ ). Helium was used as the carrier gas with a flow rate of $1.0 \mathrm{~mL} / \mathrm{min}$, and the injector temperature was set at $250{ }^{\circ} \mathrm{C}$. Compounds were identified as TMS derivatives by comparing their mass spectra with the NIST library version 1.7a and by comparing their fragmentation patterns with published data $[63,64]$. For determining the relative percentage amounts, peaks were integrated using a GC ChemStation software version C.00.01. The composition was reported as a percentage of the total peak area.

\subsection{In Vitro Antifungal Evaluation of Extracts}

The phytopathogenic fungi $R$. solani and $S$. cepivorum were provided by the Colección del Laboratorio de Patología, Departamento de Producción Agrícola (Universidad de Guadalajara). Seven-day-old strains grown on potato dextrose agar (PDA, BD Bioxon; Ciudad de Mexico, Mexico) culture medium was used. The antifungal evaluation was carried out according to the agar disk-diffusion method [65]. The 16 extracts were dissolved in $96 \%$ ethanol $(1 \mathrm{mg} / \mathrm{mL}$ ) and the $5 \mathrm{~mm}$ diameter filter paper discs (Whatman No. 1) superposed on PDA culture medium were impregnated with $10 \mu \mathrm{L}$ of each solution. Cercobin ${ }^{\circledR}$ and ethanol were used as positive and negative controls, respectively. After applying solution extracts or controls on the discs, the solvent was allowed to evaporate for $1 \mathrm{~h}$ in a laminar flow cabinet. Mycelium propagules $\left(5 \mathrm{~mm}^{3}\right)$ were inoculated on discs treated into sterile polystyrene Petri dishes (Interlux ${ }^{\circledR} 90 \times 15 \mathrm{~mm}$ ) containing $10 \mathrm{~mL}$ of PDA medium and then incubated at $28 \pm 2{ }^{\circ} \mathrm{C}$. The mean radial mycelial growth was determined by measuring the colony diameter $72 \mathrm{~h}$ after inoculation. The mean growth values were obtained and then converted into the inhibition percentage of mycelial growth $(G I)$ in relation to the control treatments using Equation (3) [65]:

$$
G I=(\%)=\left(\frac{d c-d_{t}}{d c}\right) \times 100,
$$

where $d c$ is the average diameter of the fungal colony with control and $d t$ the average diameter of fungal colony with treatments. 


\subsection{Statistical Analysis}

The data corresponding to the percentage of callus induction, dry biomass of cell suspension cultures, total phenolics and flavonoids content, and the inhibition percentage of the mycelial growth were subjected to a normality test and then an analysis of variance (ANOVA), followed by Tukey's multiple range test $(p=0.05)$. SAS 9.0 software (SAS Institute, Inc., Cary, NC, USA) was used for the statistical analysis. All experiments were conducted in triplicate.

\section{Conclusions}

In this work, the obtaining of a biotechnological culture of E. platycarpa, the phytochemical profile of cell cultures and intact plant, and its antifungal activity are reported for the first time. Although the callus induction response with auxins 2,4-D and NAA in most of the treatments was greater than $80 \%$, NAA was the most efficient auxin to establish the cell suspension culture. Cell cultures were able to produce some compounds found in wild plants, and in other cases, they produced different or larger amounts of compounds. The dichloromethane extract of CSC showed greater effectiveness at inhibiting the in vitro growth of S. cepivorum, and among these extracts, the CSC were the only ones that inhibited the growth of $R$. solani. Thus, these extracts could be a sustainable alternative resource to synthetic fungicides. Nevertheless, in future studies, research can be performed to increase the production of compounds and antifungal activity.

Supplementary Materials: The following are available online at https://www.mdpi.com/2223-774 7/10/2/414/s1, Figure S1: GC-MS chromatogram of the fatty hexane extract of sapwood, Figure S2: GC-MS chromatogram of the fatty hexane extract of heartwood, Figure S3: GC-MS chromatogram of the fatty hexane extract of leaf, Figure S4: GC-MS chromatogram of the fatty hexane extract of cell suspension cultures, Figure S5: GC-MS chromatogram of the defatted hexane extract of sapwood, Figure S6: GC-MS chromatogram of the defatted hexane extract of heartwood, Figure S7: GC-MS chromatogram of the defatted hexane extract of leaf, Figure S8: GC-MS chromatogram of the defatted hexane extract of cell suspension culture, Figure S9: GC-MS chromatogram of the dichloromethane sapwood extract, Figure S10: GC-MS chromatogram of the dichloromethane heartwood extract, Figure S11: GC-MS chromatogram of the dichloromethane leaf extract, Figure S12: GC-MS chromatogram of the dichloromethane cell suspension culture extract, Figure S13: GCMS chromatogram of the methanolic sapwood extract, Figure S14: GC-MS chromatogram of the methanolic heartwood extract, Figure S15: GC-MS chromatogram of the methanolic leaf extract, Figure S16: GC-MS chromatogram of the methanolic cell suspension cultures extract, Figure S17: Mass spectrum and structure of nonanedioic acid (azelaic acid), Figure S18: Mass spectrum and structure of hexadecanoic acid, Figure S19: Mass spectrum and structure of stigmasta-3,5-dien-7-one, Figure S20: Mass spectrum and structure of octacosanol, Figure S21: Mass spectrum and structure of $\beta$-amyrin, Figure S22: Mass spectrum and structure of octadecanoic acid, Figure S23: Mass spectrum and structure of campesterol, Figure S24: Mass spectrum and structure of stigmasterol, Figure S25: Mass spectrum and structure of $\beta$-sitosterol, Figure S26: Mass spectrum and structure of 3-phenylpropanoic acid (Benzenepropanoic acid), Figure S27: Mass spectrum and structure of $(Z, Z, Z)-9,12,15$-octadecatrienoic acid, $(Z, Z, Z)-(\alpha$-Linolenic acid), Figure S28: Mass spectrum and structure of $(Z, Z)-9,12$-octadecadienoic acid, Figure S29: Mass spectrum of D-pinitol.

Author Contributions: A.B.-A. and F.C.-S. conceived, designed, and supervised the experiments; A.S.-S., J.C.M.-C. and A.B.-A. performed the experiments and wrote a draft manuscript; A.R.-E., I.H.-D. and L.A. analyzed the data (including GC-MS) and discussed of results; A.R.-E., J.A.S.-G. and F.J.F.-T. edited and revised manuscript. All authors have read and agreed to the published version of the manuscript.

Funding: This research received no external funding.

Institutional Review Board Statement: Not applicable.

Informed Consent Statement: Not applicable. 
Data Availability Statement: The data presented in this study are available on request from the corresponding author. The data are not publicly available due to privacy.

Acknowledgments: This study was partially supported by the Consejo Nacional de Ciencia y Tecnolgía (CONACYT) during the master's degree of Alejandro Sánchez Sánchez, in the Maestría en Ciencia de Productos Forestales (PNPC-CONACYT) of the Universidad de Guadalajara. Also, we thank the Laboratorio Nacional de Estructura de Macromoléculas for the GC-MS analyses and the Prof Geogr. Roberto Zepeda Ángel for his assistance during the collection of plant material.

Conflicts of Interest: The authors declare no conflict of interest.

\section{References}

1. Silva, N.C.C.; Fernandes Júnior, A. Biological properties of medicinal plants: A review of their antimicrobial activity. J. Venom. Anim. Toxins 2010, 16, 402-413. [CrossRef]

2. $\quad$ Borges, D.F.; Lopes, E.A.; Moraes, F.A.R.; Soares, M.S.; Visôtto, L.E.; Oliveira, C.R.; Valente, V.M.M. Formulation of botanicals for the control of plant-pathogens: A review. Crop Prot. 2018, 110, 135-140. [CrossRef]

3. Mulabagal, V.; Hsin-Sheng, T. Plant cell cultures-An alternative and efficient source for the production of biologically important secondary metabolites. Int. J. Appl. Sci. Eng. 2004, 2, $29-48$.

4. McCoy, E.; O'Connor, S.E. Natural products from plant cell cultures. Prog. Drug Res. 2008, 65, 329-370. [PubMed]

5. Smetanska, I. Production of secondary metabolites using plant cell cultures. Adv. Biochem. Eng. Biotechnol. 2008, 111, 187-228.

6. Haida, Z.; Nakasha, J.J.; Hakiman, M. In vitro responses of plant growth factors on growth, yield, phenolics content and antioxidant activities of Clinacanthus nutans (Sabah Snake Grass). Plants 2020, 9, 1030. [CrossRef]

7. Rzedowsky, J.; Equihua, M. Atlas Cultural de México; De Planeta: Ciudad de México, México, 1987.

8. Martínez, M. Las Plantas Medicinales de México; Editorial Botas: Ciudad de México, México, 1996.

9. Villavicencio, N.M.A.; Pérez, E.B.E.; Ramírez, A.A. Plantas útiles del Estado de Hidalgo II; Universidad Autónoma del Estado de Hidalgo: Pachuca, Hidalgo, 2002.

10. Garcia-Campoy, A.; Garcia, E.; Muñiz-Ramirez, A. Phytochemical and Pharmacological Study of the Eysenhardtia Genus. Plants 2020, 9, 1124. [CrossRef]

11. Narváez-Mastache, J.M.; Garduño-Ramírez, M.L.; Álvarez, L.; Delgado, G. Antihyperglycemic activity and chemical constituents of Eysenhardtia platycarpa. J. Nat. Prod. 2006, 69, 1687-1691. [CrossRef] [PubMed]

12. Domínguez-Villegas, V.; Clares-Naveros, B.; García-López, M.L.; Calpena-Campmany, A.C.; Bustos-Zagal, P.; Garduño-Ramírez, M.L. Development and characterization of two nano-structured systems for topical application of flavanones isolated from Eysenhardtia platycarpa. Colloids Surf. B 2014, 116, 183-192. [CrossRef]

13. Bernabé-Antonio, A.; Maldonado-Magaña, A.; Ramírez-López, C.B.; Salcedo-Pérez, E.; Meza-Contreras, J.C.; González-García, Y.; López-Dellamary Toral, F.A.; Cruz-Sosa, F. Establishment of callus and cell suspension cultures of Eysenhardtia polystachya (Ortega) and fungistatic activity of their extracts. S. Afr. J. Bot. 2017, 112, 40-47. [CrossRef]

14. Trejo-Espino, J.L.; Rodríguez-Monroy, M.; Vernon-Carter, E.J.; Cruz-Sosa, F. Establishment and characterization of Prosopis laevigata (Humb. \& Bonpl. ex Willd) M.C. Johnst. cell suspension culture: A biotechnology approach for mesquite gum production. Acta Physiol. Plant 2011, 33, 1687-1695.

15. Maldonado-Magaña, A.; Orozco-Villafuerte, J.; Buendía-González, L.; Estrada-Zúñiga, M.E.; Bernabé-Antonio, A.; Cruz-Sosa, F. Establishment of cell suspension cultures of Prosopis laevigata (Humb. \& Bonpl. Ex Willd) M.C. Johnst to determine the effect of zinc on the uptake and accumulation of lead. Rev. Mex. Ing. Quim. 2013, 12, 489-498.

16. Su, Y.H.; Liu, Y.B.; Zhang, X.S. Auxin-cytokinin interaction regulates meristem development. Mol. Plant $2011,4,616-625$. [CrossRef]

17. Pal, A.K.; Acharya, K.; Ahuja, P.S. Endogenous auxin level is a critical determinant for in vitro adventitious shoot regeneration in potato (Solanum tuberosum L.). J. Plant Biochem. Biot. 2012, 21, 205-212. [CrossRef]

18. Machakova, I.; Zazimolova, E.; George, E.F. Plant growth regulators I: Introduction, auxins, their analogues and inhibitors. In Plant Propagation by Tissue Culture; George, E.F., Hall, M.A., De Klerk, G.J., Eds.; Springer: Dordrecht, The Netherlands, 2008; p. 175204.

19. George, E.F. Plant Tissue Culture Procedure-Background. In Plant Propagation by Tissue Culture; George, E.F., Hall, M.A., De Klerk, G.J., Eds.; Springer: Dordrecht, The Netherlands, 2008; p. 128.

20. Carmona, R.L.; Rojas, L.M.; Aura, I.U.T.; Atehortúa, G.L. Effect of nutrients starvation in the cell cycle synchronization of cell suspension cultures of Jatropha curcas L. Curr. Plant Biol. 2018, 13, $23-31$.

21. Hussein, S.; Halmi, M.I.E.; Ling, A.P.K. Modelling the growth kinetics of callus cultures from the seedling of Jatropha curcas L. according to the modified Gompertz model. J. Microbiol. Biotechn. 2016, 4, 20-23.

22. Paine, C.E.T.; Marthews, T.R.; Vogt, D.R.; Purves, D.; Rees, M.; Hector, A.; Turnbull, L.A. How to fit nonlinear plant growth models and calculate growth rates: And update for ecologists. Methods Ecol. Evol. 2012, 3, 245-256. [CrossRef]

23. Cisneros-Torres, D.; Cruz-Sosa, F.; Nicasio-Torres, M.P.; González-Cortazar, M.; Romero-Estrada, A.; Bernabé-Antonio, A. Establishment of a cell suspension culture from Calophyllum brasiliense and evaluation of its antioxidant and anti-inflammatory activity. Rev. Mex. Ing. Quim. 2020, 19, 59-70. [CrossRef] 
24. Mohamad Puad, N.I.; Abdullah, T.A. Monitoring the growth of plant cells in suspension culture. In Multifaceted Protocol in Biotechnology; Amid, A., Sulaiman, S., Jimat, D.N., Azmin, N.F., Eds.; Springer: Singapore, 2018; pp. $203-214$.

25. Benjamins, R.; Scheres, B. Auxin: The looping star in plant development. Annu. Rev. Plant Biol. 2008, 59, 443-465. [CrossRef]

26. Ammar, M.I.; Nenaah, G.E.; Mohamed, A.H.H. Antifungal activity of prenylated flavonoids isolated from Tephrosia apollinea L. against four phytopathogenic fungi. Crop Prot. 2013, 49, 21-25. [CrossRef]

27. Wang, K.; Jiang, S.; Pu, T.; Fan, L.; Su, F.; Ye, M. Antifungal activity of phenolic monoterpenes and structure related compounds against plant pathogenic fungi. Nat. Prod Res. 2019, 33, 1423-1430. [CrossRef] [PubMed]

28. Giri, L.; Dhyani, P.; Rawat, S.; Bhatt, I.D.; Nandi, S.K.; Rawal, R.S.; Pande, V. In vitro production of phenolic compounds and antioxidant activity in callus suspension cultures of Habenaria edgeworthii: A rare Himalayan medicinal orchid. Ind. Crops Prod. 2012, 39, 1-6. [CrossRef]

29. Liu, C.Z.; Saxena, P.K. Saussurea medusa cell suspension cultures for flavonoid production. In Protocols for In Vitro Cultures and Secondary Metabolite Analysis of Aromatic and Medicinal Plants; Jain, S.M., Saxena, P.K., Eds.; Springer: Dordrecht, The Netherlands, 2009; p. 5359.

30. Torres-Andrade, P.A.; Lomelí-Ramírez, M.G.; López-Dellamary, F.; Fuentes-Talavera, F.J.; Richter, H.G.; Silva-Guzmán, J.A. Natural decay resistance of Eysenhardtia polystachya (Ortega) Sarg. Int. Wood Prod. J. 2010, 1, 81-84. [CrossRef]

31. Ypushima, P.A.L. Caracterización Biológica y Química de la Madera de teca (Tectona grandis) en dos Condiciones Edafoclimáticas y su Relación con el Crecimiento. Master's Thesis, University de Guadalajara, Guadalajara, Jalisco, Mexico, 2015.

32. Santiago, M.G. Actividad Antifúngica de Extractos de las Maderas de Caesalpinia platyloba, Lysiloma latisquum, Metopium brownei y Piscidia piscipula. Master's Thesis, Universidad de Guadalajara, Guadalajara, Jalisco, Mexico, 2017.

33. Dieu-Hien, T.; Dinh, H.N.; Nhat, T.A.T.; Anh, V.B.; Tuong, H.D.; Hoang, C.N. Evaluation of the use of different solvents for phytochemical constituents, antioxidants, and in vitro anti-inflammatory activities of Severinia buxifolia. J. Food Qua. 2019, 2019, $1-9$.

34. Bukke, A.N.; Hadi, F.N.; Produtur, C.S. Comparative study of in vitro antibacterial activity of leaves, bark, heart wood and seed extracts of Caesalpinia sappan L. Asian Pac. J. Trop. Dis. 2015, 5, 903-907. [CrossRef]

35. Do, Q.D.; Angkawijaya, A.E.; Tran-Nguyen, P.L.; Huynh, L.H.; Soetaredjo, F.E.; Ismadji, S.; Ju, Y.H. Effect of extraction solvent on total phenol content, total flavonoid content, and antioxidant activity of Limnophila aromatica. J. Food Drug Anal. 2014, 22, 296-302. [CrossRef] [PubMed]

36. Arda, N.; Onay, E.; Koz, O.; Kirmizigul, S. Monosaccharides and polyols from mistletoes (Viscum album L.) growing on two different host species. Biologia (Bratislava) 2003, 58, 1037-1042.

37. Wink, M. Evolution of secondary metabolites in legumes (Fabaceae). S. Afr. J. Bot. 2013, 89, 164-175. [CrossRef]

38. Musa, A.M.; Ibrahim, M.A.; Aliyu, A.B.; Abdullahi, M.S.; Tajuddeen, N.; Ibrahim, H.; Oyewale, A.O. Chemical composition and antimicrobial activity of hexane leaf extract of Anisopus mannii (Asclepiadaceae). J. Intercul. Ethnopharmacol. 2015, 4, 129-133. [CrossRef]

39. Hosseinihashemi, S.K.; Safdari, V.; Kanani, S. Comparative chemical composition of n-hexane and ethanol extractives from the heartwood of black locust. Asian J. Chem. 2013, 25, 929-933. [CrossRef]

40. Miranda, I.; Sousa, V.; Ferreira, J.; Pereira, H. Chemical characterization and extractives composition of heartwood and sapwood from Quercus faginea. PLoS ONE 2017, 12, e0179268. [CrossRef] [PubMed]

41. Pérez-González, M.Z.; Nieto-Trujillo, A.; Gutiérrez-Rebolledo, G.A.; García-Martínez, I.; Estrada-Zúñiga, M.E.; Bernabé-Antonio, A.; Cruz-Sosa, F. Lupeol acetate production and antioxidant activity of a cell suspension culture from Cnidoscolus chayamansa leaves. S. Afr. J. Bot. 2019, 125, 30-38. [CrossRef]

42. Regasini, L.O.; Vieira-Júnior, G.M.; Fernandes, D.C.; Da Silva Bolzani, V.; Cavalheiro, A.J.; Siqueira Silva, D.H. Identification of triterpenes and sterols from Pterogyne nitens (Fabaceae-caesalpinioideae) using high-resolution gas chromatography. J. Chil. Chem. Soc. 2009, 54, 218-221. [CrossRef]

43. Meela, M.M.; Mdee, L.K.; Masoko, P.; Eloff, J.N. Acetone leaf extracts of seven invasive weeds have promising activity against eight important plant fungal pathogens. S. Afr. J. Bot. 2019, 121, 442-446. [CrossRef]

44. Bhagwat, M.K.; Datar, A.G. Antifungal activity of herbal extracts against plant pathogenic fungi. Arch. Phytopathol. Plant Prot. 2014, 47, 959-965. [CrossRef]

45. Liu, S.; Ruan, W.; Li, J.; Xu, H.; Wang, J.; Gao, Y.; Wang, J. Biological control of phytopathogenic fungi by fatty acids. Mycopathologia 2008, 166, 93-102. [CrossRef]

46. Gołębiowski, M.; Cerkowniak, M.; Boguś, M.I.; Włóka, E.; Dawgul, M.; Kamysz, W.; Stepnowski, P. Free fatty acids in the cuticular and internal lipids of Calliphora vomitoria and their antimicrobial activity. J. Insect Physiol. 2013, 59, 416-429. [CrossRef]

47. Bergsson, G.; Arnfinnsson, J.; Steingrímsson, Ó.; Thormar, H. In vitro killing of Candida albicans by fatty acids and monoglycerides. Antimicrob. Agents Chemother. 2001, 45, 3209-3212. [CrossRef]

48. Li, X.C.; Jacob, M.R.; ElSohly, H.N.; Nagle, D.G.; Smillie, T.J.; Walker, L.A.; Clark, A.M. Acetylenic acids inhibiting Azole-resistant Candida albicans from Pentagonia gigantifolia. J. Nat. Prod. 2003, 66, 1132-1135. [CrossRef] [PubMed]

49. Pohl, C.H.; Kock, J.L.; Thibane, V.S. Antifungal free fatty acids: A review. Sci. Microb. Pathog. Commun. Curr. Res. Technol. Adv. 2011, 1, 61-71.

50. Calvo-Garrido, C.; Elmer, P.A.; Parry, F.J.; Viñas, I.; Usall, J.; Torres, R.; Agnew, R.H.; Teixidó, N. Mode of action of a fatty acid-based natural product to control Botrytis cinerea in grapes. J. Appl. Microbiol. 2014, 116, 967-979. [CrossRef] 
51. Ueda, T.; Kai, H.; Taniguchi, E. Growth inhibition of soil-borne pathogenic fungi by typical sterols. Soil Biol. Biochem. 1990, 22, 987-991. [CrossRef]

52. Choi, N.H.; Jang, J.Y.; Choi, G.J.; Choi, Y.H.; Jang, K.S.; Min, B.S.; Dang, Q.L.; Kim, J.C. Antifungal activity of sterols and dipsacus saponins isolated from Dipsacus asper roots against phytopathogenic fungi. Pestic. Biochem. Physiol. 2017, 141, 103-108. [CrossRef] [PubMed]

53. Yenjit, P.; Issarakraisila, M.; Intana, W.; Chantrapromma, K. Fungicidal activity of compounds extracted from the pericarp of Areca catechu against Colletotrichum gloeosporioides in vitro and in mango fruit. Postharvest Biol. Technol. 2010, 55, 129-132. [CrossRef]

54. Canzi, E.F.; Marques, F.A.; Teixeira, S.D.; Tozzi, A.M.G.A.; Silva, M.J.; Duarte, R.M.T.; Duarte, M.C.T.; Ruiz, A.L.T.G.; Monteiro, P.A.; de Carvalho, J.E.; et al. Prenylated flavonoids from roots of Dahlstedtia glaziovii (Fabaceae). J. Braz. Chem. Soc. 2014, 25, 995-1001.

55. Cursino, L.M.C.; Lima, N.M.; Murillo, R.; Nunez, C.V.; Merfort, I.; Humar, M. Isolation of flavonoids from Deguelia duckeana and their effect on cellular viability, AMPK, eEF2, eIF2 and eIF4E. Molecules 2016, 21, 192. [CrossRef] [PubMed]

56. De Lucca, A.J. Harmful fungi in both agriculture and medicine. Rev. Iberoam. Micol. 2007, 24, 3-13. [CrossRef]

57. Murashige, T.; Skoog, F. A revised medium for rapid growth and bio assays with tobacco tissue cultures. Physiol. Plant. 1962, 15, 473-497. [CrossRef]

58. Nicasio-Torres, M.P.; Pérez-Hernández, J.; González-Cortazar, M.; Meckes-Fischer, M.; Tortoriello, J.; Cruz-Sosa, F. Production of potential anti-inflammatory compounds in cell suspension cultures of Sphaeralcea angustifolia (Cav.) G. Don. Acta Physiol. Plant. 2016, 38, 209. [CrossRef]

59. Zwietering, M.H.; Jongenburger, I.; Rombouts, F.M.; van't Riet, K. Modeling of the bacterial growth curve. Appl. Environ. Microbiol. 1990, 56, 1875-1881. [CrossRef]

60. DuBois, M.; Gilles, K.A.; Hamilton, J.K.; Rebers, P.A.; Smith, F. Colorimetric method for determination of sugars and related substances. Anal. Chem. 1956, 28, 350-356. [CrossRef]

61. Wu, T.T.; Shiau, S.Y.; Chang, R.C. Effects of drying temperature and extraction solvent on the antioxidant, color and sensory properties of Angelica sinensis leaves. J. Food Process. Preserv. 2013, 37, 371-379. [CrossRef]

62. Liu, M.; Li, X.Q.; Weber, C.; Lee, C.Y.; Brown, J.; Liu, R.H. Antioxidant and antiproliferative activities of raspberries. J. Agric. Food Chem. 2002, 50, 2926-2930. [CrossRef] [PubMed]

63. Alonso-Castro, A.J.; Zapata-Morales, J.R.; Arana-Argáez, V.; Torres-Romero, J.C.; Ramírez-Villanueva, E.; Pérez-Medina, S.E.; Ramírez-Morales, M.A.; Juárez-Méndez, M.A.; Infante-Barrios, Y.P.; Martínez-Gutiérrez, F.; et al. Pharmacological and toxicological study of a chemical-standardized ethanol extract of the branches and leaves from Eysenhardtia polystachya (Ortega) Sarg. (Fabaceae). J. Ethnopharmacol. 2018, 224, 314-322. [CrossRef]

64. Garland, S.; Goheen, S.; Donald, P.; McDonald, L.; Campbell, J. Application of derivatization gas chromatography/mass spectrometry for the identification and quantitation of pinitol in plant roots. Anal. Lett. 2009, 42, 2096-2105. [CrossRef]

65. Amadioha, A.C. Controlling rice blast in vitro and in vivo with extracts of Azadirachta indica. Crop Prot. 2000, 19, 287-290. [CrossRef] 